\title{
Statistical mechanics of quasi-geostrophic flows on a rotating sphere.
}

\author{
C.Herbert $^{1,2}$, B.Dubrulle ${ }^{1}$, P.H.Chavanis ${ }^{3}$ and D.Paillard ${ }^{2}$ \\ ${ }^{1}$ Service de Physique de l'Etat Condensé, DSM, CEA Saclay, CNRS URA 2464, \\ Gif-sur-Yvette, France \\ ${ }^{2}$ Laboratoire des Sciences du Climat et de l'Environnement, IPSL, \\ CEA-CNRS-UVSQ, UMR 8212, Gif-sur-Yvette, France \\ ${ }^{3}$ Laboratoire de Physique Théorique (IRSAMC), CNRS and UPS, Université de \\ Toulouse, 31062 Toulouse, France \\ E-mail: corentin.herbert@lsce.ipsl.fr
}




\begin{abstract}
.
Statistical mechanics provides an elegant explanation to the appearance of coherent structures in two-dimensional inviscid turbulence: while the fine-grained vorticity field, described by the Euler equation, becomes more and more filamented through time, its dynamical evolution is constrained by some global conservation laws (energy, Casimir invariants). As a consequence, the coarse-grained vorticity field can be predicted through standard statistical mechanics arguments (relying on the Hamiltonian structure of the two-dimensional Euler flow), for any given set of the integral constraints.

It has been suggested that the theory applies equally well to geophysical turbulence; specifically in the case of the quasi-geostrophic equations, with potential vorticity playing the role of the advected quantity. In this study, we demonstrate analytically that the Miller-Robert-Sommeria theory leads to non-trivial statistical equilibria for quasi-geostrophic flows on a rotating sphere, with or without bottom topography. We first consider flows without bottom topography and with an infinite Rossby deformation radius, with and without conservation of angular momentum. When the conservation of angular momentum is taken into account, we report a case of second order phase transition associated with spontaneous symmetry breaking. In a second step, we treat the general case of a flow with an arbitrary bottom topography and a finite Rossby deformation radius. Previous studies were restricted to flows in a planar domain with fixed or periodic boundary conditions with a beta-effect.

In these different cases, we are able to classify the statistical equilibria for the largescale flow through their sole macroscopic features. We build the phase diagrams of the system and discuss the relations of the various statistical ensembles.
\end{abstract}

Keywords: Classical phase transitions (Theory), Phase diagrams (Theory), Metastable states, Turbulence

Submitted to: Journal of Statistical Mechanics: Theory and Experiments.

\title{
Contents
}

1 Introduction 4

2 Statistical Mechanics of the quasi-geostrophic equations 6

2.1 Definitions and notations . . . . . . . . . . . . . . 6

2.2 The quasi-geostrophic equations . . . . . . . . . . . . . . 7

2.3 Maximum entropy states . . . . . . . . . . . . . . . . . 8

2.4 The linear $q-\psi$ relationship . . . . . . . . . . . . . . . . . . . . 10

2.5 Statistical ensembles and variational problems . . . . . . . . . 12

3 The equilibrium mean flow in the barotropic case $(R=\infty)$ without bottom topography $\left(h_{B}=0\right) \quad[14$

3.1 Fixed energy and circulation . . . . . . . . . . . . . 14

3.1.1 Case $\beta \notin \operatorname{Sp} \Delta$ : the continuum solution . . . . . . . . 15

3.1 .2 Case $\beta \in \operatorname{Sp} \Delta \ldots \ldots \ldots \ldots$. . . . . . . . . . . . . . . . 
3.1.3 Nature and stability of the critical points . . . . . . . . . . 18

3.1.4 Summary of the results . . . . . . . . . . . . . . 19

3.2 Fixed energy, circulation and angular momentum . . . . . . . . . . 19

3.2.1 Case $\beta \notin \operatorname{Sp} \Delta$ : the continuum solution . . . . . . . . . . 20 20

3.2 .2 Case $\beta \in \operatorname{Sp} \Delta \ldots \ldots \ldots \ldots \ldots 21$

3.2 .3 Case $\beta=\beta_{1} \ldots \ldots \ldots \ldots \ldots 22$

3.2.4 Nature and stability of the critical points . . . . . . . . . . . 23

3.2 .5 Summary of the results . . . . . . . . . . . . . . 24 24

3.2.6 Discussion of the ensemble equivalence properties . . . . . . . . 29 29

4 General case: quasi-geostrophic flow over a topography. 30

4.1 The general mean field equation and its solution . . . . . . . . . . . . . 30

4.2 Degenerate solutions of the mean field equation . . . . . . . . . . . 32

4.3 Stability of the statistical equilibria . . . . . . . . . . . . . . . . . . . . . . . . . .

4.4 The effect of the bottom topography . . . . . . . . . . . . . . . 34

4.5 The role of the Rossby deformation radius . . . . . . . . . . . . 39

5 Discussion 40

6 Conclusion 42

Appolidibodly rotations 43

Appkindixa Rn energy for a flow with given angular momentum 44 


\section{Introduction}

An important characteristic of two-dimensional turbulent fluid flows is the emergence of coherent structures: in the 80s, numerical simulations [1, 2] showed that a turbulent flow tends to organize itself spontaneously into large-scale coherent vortices for a wide range of initial conditions and parameters. Laboratory experiments reported similar observations [3, 4, 5, 6]. Large-scale coherent structures are also ubiquitous in planetary atmospheres and in oceanography. Due to the long-lived nature of these structures, it is very appealing to try to understand the reasons for their appearance and maintenance through a statistical theory.

This endeavour is supported by theoretical arguments: as first noticed by Kirchhoff, the equations for a perfect fluid flow can be recast in a Hamiltonian form, which makes them a priori suitable for standard statistical mechanics treatments, as a Liouville theorem automatically holds. The first attempt along these lines was Onsager's statistical theory of point vortices [7]. One peculiar outcome of Onsager's theory is the appearance of negative temperature states at which large-scale vortices form $\$$. The point vortex theory was further developed by many authors [8, 9, 10, 11, 12, 13] and its relations with plasma physics [14, 8, 15] and astrophysics [16, 17] was pointed out. The main problem with Onsager's theory is that it describes a finite collection of point vortices and not a continuous vorticity field. In particular, some invariant quantities of perfect fluid flow are singular in the point vortex description, and it is not easy to construct a continuum theory as a limit of the point-vortex theory.

Subsequent attempts essentially considered truncations of the equations of motion in the spectral space. Lee [18] obtained a Liouville theorem in the spectral phase space and constructed a statistical theory taking into account only the conservation of energy. Kraichnan built a theory on the basis of the conservation of the quadratic invariants: energy and enstrophy [19, 20]. The theory mainly predicts an equilibrium energy spectrum corresponding to an equipartition distribution. This spectrum has been extensively confronted with experiments and numerical simulations (e.g. [21, 22]) but the discussion remains open [23].

More recently, Miller [24, 25] and Robert \& Sommeria [26, 27] independently developed a theory for the continuous vorticity fields, taking into account all the invariants of motion. Due to the infinite number of these invariants, the rigorous mathematical justification is more elaborate than previous approaches and relies on convergence theorems for Young measures [28, 29]. Miller [25] provides two alternative derivations, perhaps more heuristic, the first one being based on phase space-counting ideas similar to Boltzmann's classical equilibrium statistical mechanics (see also LyndenBell [30]), while the second one uses a Kac-Hubbard-Stratonovich transformation. The MRS theory was checked against laboratory experiments [31] and numerical studies

$\ddagger$ Technically, the existence of negative temperatures results from the fact that the coordinates $x$ and $y$ of the point vortices are canonically conjugate. This implies that the phase space coincides with the configuration space, so it is finite. This leads to negative temperature states at high energies. 
[32, 33] in a wide variety of publications [34].

One of the main interest of applying statistical mechanical theories to inviscid fluid flows is that it provides a very powerful tool to investigate directly the structure of the final state of the flow, regardless of the temporal evolution that leads to this final state. From a practical point of view, such a tool would of course be of great value as it is well-known that turbulence simulations are very greedy in terms of computational resources. In some rare cases, computations can be carried out analytically and it is even possible to elucidate the final organization of the flow directly from the mean field equations obtained from statistical mechanics. In any case, the interest is also theoretical since equilibrium statistical mechanics of inviscid fluid flows can be seen as a specific example of long-range interacting systems [35], whose statistical mechanics is known to yield peculiar behaviors, in phase transitions and ensemble inequivalence [36, 37, 38, 39, 40, 41]. As an example, statistical mechanics provided valuable insight in the understanding of a von Karman experiment, in particular in transitions between different flow regimes [42, 43], fluctuation-dissipation relations [44] and Beltramization [45, 46, 31, 47].

One particular area where avoiding long numerical turbulence simulations would be highly beneficial is geophysics. Jupiter's great red spot provides a prototypical example of application of statistical mechanics to geophysical fluid dynamics [25, 48, 49, 50, 51], in which valuable insight is gained from the statistical theory. Even before, the Kraichnan energy-enstrophy theory was extensively used to discuss energy and enstrophy spectra in the atmosphere [52, 53] and topographic turbulence [54, 55]. However, only one study [56] considers the global equilibrium flow in a spherical geometry, with encouraging results, but this study does not investigate the structure of the flow in a systematic way. Statistical mechanics of the continuous vorticity field conserving all the invariants has also been applied to the Earth's oceans, focusing either on small-scale parameterizations [57, 58, 59, 60, 61] or on meso-scale structures [62] (and in particular the Fofonoff flow [39, 40, 63]).

In this study, we investigate analytically the statistical equilibria of the large-scale general circulation of the Earth's atmosphere, modelled by the quasigeostrophic equations, taking into account the spherical geometry (with possible bottom topography) and the full effect of rotation, in the framework of the MRS theory. More precisely, we show that in the absence of a bottom topography, due to the spherical geometry, the solution to the statistical mechanics problem can be derived in a very simple way. The result is, however, highly non trivial because, when the conservation of angular momentum is properly accounted for, it leads to a second order phase transition associated with a spontaneous symmetry breaking. Since all the previous studies used a $\beta$-effect instead of the full Coriolis parameter and focused on rectangular bounded regions rather than on the full sphere, this simple solution was not noticed before. We draw the phase diagrams of the system in both microcanonical and grandcanonical ensembles. The relations between the two statistical ensembles is described in detail and we present a refined notion of marginal equivalence of ensemble (see also 
[64]). In the presence of a bottom topography, we obtain semi-persistent equilibria reminiscent of the structures observed in the atmosphere. They correspond to saddle points of entropy. Strictly speaking, they are unstable since they can be destabilized by certain infinitesimal perturbations belonging to particular subspaces of the dynamical space: for these saddle points of the entropy surface, there is at least one direction along which the entropy increases while the constraints remain satisfied. However, it may take a long time before the system spontaneously generates these perturbations. Therefore, these states may persist for a long time before finally being destabilized [65]. In the atmospheric context, these semi-persistent equilibrium states could account for situations of atmospheric blocking where a large scale structure can form for a few days before finally disappearing.

In section 2, we present the general statistical mechanics of the quasi-geostrophic equations. In section 3 we obtain the structure of the equilibrium mean flow in the particular case of a sphere without bottom topography in the limit of infinite Rossby deformation radius, with and without conservation of angular momentum. In section 4 , we examine the effect of the bottom topography and of the Rossby deformation radius. Section 5 presents a discussion of the obtained results and a comparison with previously published results, while conclusions are presented in section 6 .

\section{Statistical Mechanics of the quasi-geostrophic equations}

\subsection{Definitions and notations}

We consider here an incompressible, inviscid, fluid on the two dimensional sphere $S^{2}$ (denoted $D$ to keep notations simple). The coordinates are $(\theta, \phi)$ where $\theta \in[0, \pi]$ is the polar angle (the latitude is thus $\pi / 2-\theta$ ) and $\phi \in[0,2 \pi]$ the azimuthal angle. In the following, for any quantity $A$, we note $\langle A\rangle$ its average value over the whole domain:

$$
\langle A\rangle=\frac{\int_{D} A(\mathbf{r}) d^{2} \mathbf{r}}{\int_{D} d^{2} \mathbf{r}} .
$$

We introduce the eigenvectors of the Laplacian $\Delta$ on the sphere. These are the spherical harmonics $Y_{n m}$ with eigenvalues $\beta_{n}$ :

$$
\begin{aligned}
Y_{n m}(\theta, \phi) & =\sqrt{\frac{2 n+1}{4 \pi} \frac{(n-m) !}{(n+m) !}} P_{n}^{m}(\cos \theta) e^{i m \phi}, \\
\Delta Y_{n m} & =\beta_{n} Y_{n m},
\end{aligned}
$$

where $P_{n}^{m}$ are the associated Legendre polynomials and $\beta_{n}=-n(n+1)$ [66]. The scalar product on the vector space of complex-valued functions on the sphere $S^{2}$ is defined as usual as

$$
\langle f \mid g\rangle=\int_{0}^{2 \pi} d \phi \int_{0}^{\pi} d \theta \sin \theta \overline{f(\theta, \phi)} g(\theta, \phi),
$$

where the bar denotes complex conjugaison, so that the spherical harmonics form an orthonormal basis of the Hilbert space $L^{2}\left(S^{2}\right)$ :

$$
\left\langle Y_{n m} \mid Y_{p q}\right\rangle=\delta_{n p} \delta_{m q} .
$$


Note that $\langle f \mid g\rangle=4 \pi\langle\bar{f} g\rangle$.

For applications to the Earth, we shall take the inverse of the Earth's rotation rate $\Omega$ as the time unit and we set $\tilde{r}=r / R_{T}$ in the radial direction so that the Earth mean radius $R_{T}$ is the length unit. Hence all the analytical calculations are carried out on the unit sphere $S^{2}$, while we retain the $\Omega$ dependance in the calculations to stress the effect of rotation in the formulae, even though for numerical applications, we will always take $\Omega=1$.

\subsection{The quasi-geostrophic equations}

We consider here the simplest model for geophysical flows: the one-layer quasigeostrophic equations, also called the equivalent barotropic vorticity equations. We assume that the velocity field $\mathbf{v}$ satisfies the incompressibility condition $\nabla \cdot \mathbf{v}=0$, so that we can introduce a stream function $\psi$ such that $\mathbf{v}=-\hat{\mathbf{r}} \times \nabla \psi$, and define the potential vorticity as

$$
q=-\Delta \psi+h+\frac{\psi}{R^{2}}
$$

where $h$ is the topography and $R$ the Rossby deformation radius [67]. The evolution of the potential vorticity is given by the quasi-geostrophic equation

$$
\partial_{t} q+\mathbf{v} \cdot \nabla q=0 .
$$

In other words, the flow conserves potential vorticity. Together with the fact that the flow is incompressible, this implies the conservation of the integral of any function of potential vorticity $I_{g}=\int_{D} g(q) d^{2} \mathbf{r}$, called Casimir invariants ( $g$ being an arbitrary function). In particular, any moment $\Gamma_{n}=\int_{D} q^{n} d^{2} \mathbf{r}$ of the potential vorticity is conserved. $\Gamma_{1}$ will be called here the circulation and $\Gamma_{2}$ the potential enstrophy. The energy, given by

$$
E=\frac{1}{2} \int_{D}(q-h) \psi d^{2} \mathbf{r}=\frac{1}{2} \int_{D}\left((\nabla \psi)^{2}+\frac{\psi^{2}}{R^{2}}\right) d^{2} \mathbf{r}
$$

is also conserved. Finally, due to the spherical symmetry, one may also consider a supplementary invariant: the integral over the domain of the vertical component of angular momentum

$$
L=\int_{D} u \sin \theta d^{2} \mathbf{r}=\int_{D} q \cos \theta d^{2} \mathbf{r},
$$

where $u=-\partial_{\theta} \psi$ is the zonal component of velocity. In Appendix A, we show that, for a solid-body rotation, the dynamical invariants $E$ and $L$ are not independent: they obey a relation of the form $E=E^{*}(L)$, with $E^{*}(L)=3 L^{2} / 4$. We also show Appendix B that, for any flow, $E \geq E^{*}(L)$.

For fluid motion on a rotating sphere, the term $h$ includes the Coriolis parameter $f=2 \Omega \cos \theta$. Following [56], the general form we will consider here is $h=f+f \frac{h_{B}}{H_{A}}$ with $h_{B}$ the bottom topography and $H_{A}$ the average height of the fluid. The relative vorticity is $\omega=-\Delta \psi$ and the absolute vorticity $\omega+f$. In the limit of infinite Rossby 
deformation radius $(R=\infty)$ and no topography $(h=0)$, we recover the 2D Euler equations. Introducing the Poisson brackets on the sphere

$$
\{A, B\}=\frac{1}{r^{2} \sin \theta}\left(\frac{\partial A}{\partial \phi} \frac{\partial B}{\partial \theta}-\frac{\partial A}{\partial \theta} \frac{\partial B}{\partial \phi}\right)
$$

the quasi-geostrophic equation (7) reads

$$
\partial_{t} q+\{q, \psi\}=0 \text {. }
$$

It is well known in the case of the Euler (or quasi-geostrophic in a planar domain) equations that the Poisson bracket form implies that the steady states of the equations correspond to $q=F(\psi)$ with $F$ an arbitrary function. In fact, due to the particular geometry considered here, the form of the steady-states must be slightly refined. Let us consider solutions of the quasi-geostrophic equations of the form $q(\theta, \phi, t)=q\left(\theta, \phi-\Omega_{L} t\right)$. Substituting this relation into equation (11), we obtain

$$
-\Omega_{L} \frac{\partial q}{\partial \phi}+\{q, \psi\}=\left\{q, \Omega_{L} \cos \theta\right\}+\{q, \psi\}=0,
$$

so that $q=F\left(\psi+\Omega_{L} \cos \theta\right)$, with $F$ an arbitrary function. This is the general form of the solutions of the quasi-geostrophic equations which are stationary in a frame rotating with angular velocity $\Omega_{L}$ with respect to the initial reference frame (which rotates with angular velocity $\Omega$ ). When $\Omega_{L}=0$, we recover the previous $q-\psi$ relationship. However, due to the spherical symmetry, there is no reason to select the reference frame $\Omega_{L}=0$ a priori.

In the next section, we show that statistical mechanics allows to select a particular function $F$ on the grounds that it is the most probable equilibrium state respecting the constraints.

\subsection{Maximum entropy states}

If we were to inject a droplet of dye in a turbulent two-dimensional flow, we would observe a complex mixing where the originally regular patch of dye turns into finer and finer filaments as time goes on. After a while, the filaments are so intertwined that the dye seems homogeneously distributed over the fluid to a human eye: the coarsegrained dye concentration is homogeneous. In the quasi-geostrophic equations, it is potential vorticity that is mixed by the flow (equation (7)). The crucial difference is that the advected quantity is no longer a passive tracer but plays an active role in the dynamics. Due to the conservation constraints associated to the quasi-geostrophic equations, the potential vorticity mixing will not lead to an homogeneous coarsegrained distribution. In particular, the energy constaint prevents complete mixing. We wish to determine what this final coarse-grained state will be, regardless of the details of the fine-grained structure of the potential vorticity field. Analogously to classical statistical mechanics [68, 69, 70], after identifying the correct description for microstates (exact fine-grained vorticity field) and macrostates (the coarse-grained vorticity field, mathematically represented as a Young measure), one selects the 
macrostate that maximizes the statistical entropy subject to the relevant macroscopic constraints (conserved quantities), as developed by Miller and Robert [24, 25, 26, 27]. The underlying fondamental property is that an overwhelming majority of microstates lie in the vicinity of the equilibrium macrostate. The implicit separation of scales between microstates and macrostates implies that the contributions of the smallscale fluctuations of vorticity are discarded in the macroscopic quantities (strictly speaking, this is true for the energy but not for the Casimirs: computing the moments of the vorticity distribution using the fine-grained distribution or the coarse-grained distribution yields different results). As a consequence, the Miller-Robert-Sommeria (MRS) theory is a mean-field theory [71, 25]. Note also that albeit all the dynamically conserved quantities of the equations are imposed as constraints in the statistical mechanics, the topological constraints are not conserved: a connected vorticity domain should remain connected through time, while in the statistical mechanics the only thing that is conserved is the area of this domain.

At the microscopic level, the potential vorticity is fully determined by the initial conditions and the evolution equation (7). At the macroscopic level, we consider the coarse-grained potential vorticity $q$ as a random variable with probability distribution $\rho$ : the probability that the potential vorticity has the value $\sigma$ with an error $d \sigma$ at point $\mathbf{r}$ is $\rho(\mathbf{r}, \sigma) d \sigma$. The potential vorticity distribution $\rho(\mathbf{r}, \sigma)$ characterizes the macroscopic state. The potential vorticity distribution must satisfy the normalization condition $\int \rho(\mathbf{r}, \sigma) d \sigma=1$ at each point of the domain, and the mean value of the potential vorticity is given by $\bar{q}=\int \rho(\mathbf{r}, \sigma) \sigma d \sigma$. We introduce a stream function $\psi$ corresponding to the ensemble-mean potential vorticity through $\bar{q}=-\Delta \psi+\frac{\psi}{R^{2}}+h$. The statistical entropy of the probability distribution $\rho$ is

$$
S[\rho]=-\operatorname{Tr}(\rho \ln \rho)=-\int_{-\infty}^{+\infty} d \sigma \int_{D} d^{2} \mathbf{r} \rho(\mathbf{r}, \sigma) \ln \rho(\mathbf{r}, \sigma) .
$$

We are looking for the probability distribution $\rho$ that maximizes the statistical entropy functional $S[\rho]$ subject to the constraints mentioned in section 2.2 global conservation of energy and Casimir functionals. The conservation of all the Casimirs is equivalent to the conservation of the area of each potential vorticity level $\gamma(\sigma)=\int_{D} \rho(\mathbf{r}, \sigma) d^{2} \mathbf{r}$. Hence the statistical equilibria must satisfy

$$
\begin{aligned}
\delta S-\tilde{\beta} \delta E-\int & \tilde{\alpha}(\sigma) \delta \gamma(\sigma) d \sigma-\tilde{\mu} \int_{D} \delta\left(\int \sigma \rho(\mathbf{r}, \sigma) d \sigma\right) \cos \theta d^{2} \mathbf{r} \\
& -\int_{D} \zeta(\mathbf{r}) \delta\left(\int \rho(\mathbf{r}, \sigma) d \sigma\right) d^{2} \mathbf{r}=0
\end{aligned}
$$

where $\tilde{\beta}, \tilde{\alpha}(\sigma), \tilde{\mu}$ and $\zeta(\mathbf{r})$ are respectively the Lagrange multipliers associated with the conservation of energy, potential vorticity levels, angular momentum, and normalization.

The resulting potential vorticity probability density is the Gibbs state

$$
\rho(\mathbf{r}, \sigma)=\frac{1}{Z} g(\sigma) e^{-\tilde{\beta} \sigma \psi-\tilde{\mu} \sigma \cos \theta},
$$


where $g(\sigma)=e^{-\tilde{\alpha}(\sigma)}$ and $Z=e^{1+\zeta(\mathbf{r})}$. Due to the normalization condition, the partition function $Z$ is also given by

$$
Z=\int g(\sigma) e^{-\tilde{\beta} \sigma \psi-\tilde{\mu} \sigma \cos \theta} d \sigma
$$

and the ensemble-mean potential vorticity satisfies the usual relation

$$
\bar{q}=-\frac{1}{\tilde{\beta}} \frac{\partial \ln Z}{\partial \psi} .
$$

The right-hand side of this equation is a certain function $F$ of the relative stream function $\psi_{*}=\psi+\frac{\tilde{\mu}}{\tilde{\beta}} \cos \theta$. Hence, for given values of the Lagrange multipliers $\tilde{\alpha}$ and $\tilde{\beta}$, the statistical entropy maximization procedure selects a functional relationship between potential vorticity and relative stream function at steady-state: $\bar{q}=F_{\tilde{\beta}, \tilde{\alpha}, \tilde{\mu}}\left(\psi+\frac{\tilde{\mu}}{\tilde{\beta}} \cos \theta\right)$. This describes a flow rotating with angular velocity $\Omega_{L}=\tilde{\mu} / \tilde{\beta}$ with respect to the terrestrial frame. Therefore, statistical mechanics selects steady states of the QG equations in a rotating frame. The resulting mean field equation is simply

$$
-\Delta \psi+\frac{\psi}{R^{2}}+h=F\left(\psi+\frac{\tilde{\mu}}{\tilde{\beta}} \cos \theta\right) .
$$

This is the general mean field equation for equilibrium states of the quasi-geostrophic equations. In the limit $R \rightarrow \infty, h=0, \tilde{\mu}=0$, one recovers the well-known mean field equation for the Euler equation. Note that in the case of only two potential vorticity levels $\sigma_{1}$ and $\sigma_{-1}$, the partition function $Z$ is simply $Z=g\left(\sigma_{1}\right) e^{-\tilde{\beta} \sigma_{1} \psi_{*}}+g\left(\sigma_{-1}\right) e^{-\tilde{\beta} \sigma_{-1} \psi_{*}}$, so that after straightforward computations, we find the $q-\psi$ relationship $\bar{q}=B-$ $A \tanh \left(\alpha+A \tilde{\beta} \psi_{*}\right)$ with $e^{-2 \alpha}=\frac{g\left(\sigma_{1}\right)}{g\left(\sigma_{-1}\right)}, B=\frac{\sigma_{1}+\sigma_{-1}}{2}, A=\frac{\sigma_{1}-\sigma_{-1}}{2}$. With $A=1$ and $\tilde{\beta}=-\frac{C}{R^{2}}$, we recover the $q-\psi$ relationship [50]:

$$
\bar{q}=B-\tanh \left(\alpha-\frac{C \psi_{*}}{R^{2}}\right) .
$$

To determine the statistical equilibrium state, we have to solve the mean field equation (17), relate the Lagrange multipliers to the constraints and study the stability of the solutions (whether they are entropy maxima or saddle points). If several entropy maxima are found for the same parameters (conserved quantities), we must distinguish metastable states (local entropy maxima) from fully stable states (global entropy maxima).

\subsection{The linear $q-\psi$ relationship}

In practice, the mean field equation (17) is difficult to solve because the function $F$ is in general nonlinear due to the conservation of all the moments of the fine-grained potential vorticity. Besides, it is generally difficult to relate the Lagrange multipliers to the conserved quantitites. The two levels system of [50] provides an example of a case where it is possible to write down explicitly the $q-\psi$ relationship but the meanfield equation 18 is not analytically solvable without further approximations. Nevertheless, 
efficient numerical methods do exist, like for instance the algorithm of Turkington and Whitaker [72] or the method of relaxation equations [73, 74].

To go further with analytical methods, a common solution is to linearize the $q-\psi$ relationship. Several justifications of this procedure can be given, which can be grossly classified in two types of approaches. In the first approach, one simply discards the effect of the high-order fine-grained potential vorticity moments (it would be possible to include them one by one hierarchically), while in the second approach, their effect is prescribed through a gaussian prior distribution for small-scale potential vorticity (in the general theory, one can specify a non-gaussian prior, which would lead to a nonlinear $q-\psi$ relationship).

- In the limit of strong mixing $\tilde{\beta} \sigma \psi \ll 1$, the argument of the exponential in the partition function is small and a power expansion of $Z$ can be carried out. The rigorous computation is presented in [75] and yields a linear $\bar{q}-\psi$ relationship. This power series expansion can be done at virtually any order. At first-order, equation (17) becomes identical to the mean field equation obtained by minimizing the coarse-grained enstrophy $\Gamma_{2}^{c g}=\int_{D} \bar{q}^{2} d^{2} \mathbf{r}$ with fixed energy, circulation and angular momentum. The value of the fine-grained enstrophy $\Gamma_{2}^{f g}=\int_{D} \overline{q^{2}} d^{2} \mathbf{r}$ is fixed by the initial condition and we always have $\Gamma_{2}^{c g} \leq \Gamma_{2}^{f g}$. The strong mixing limit thus corresponds to cases where the energy, circulation, angular momentum (called robust invariants because they are expressed in terms of the coarse-grained potential vorticity) and fine-grained enstrophy are the only important invariants and the higher-order moments of the fine-grained enstrophy (called fragile invariants) do not play any role. This can be seen as a form of justification in the framework of statistical mechanics of inviscid fluids of the early phenomenological minimum enstrophy principle suggested by [76], [77] and [78] on the basis of the inverse cascade of Batchelor [79] for finite viscosities. The connection between the inviscid statistical theory and the phenomenological selective decay approach is discussed at length in [75], [80] and [81].

- For any given energy, one can find a vorticity level distribution $\gamma(\sigma)$ such that the function $F$ is linear. This corresponds to a gaussian $g(\sigma)$ (see [25]). Indeed, if $g(\sigma)=\frac{1}{\sqrt{2 \pi \eta}} e^{-\frac{\left(\sigma-\sigma_{m}\right)^{2}}{2 \eta}}$ is a Gaussian with mean value $\sigma_{m}$ and standard deviation $\eta$, the analytical computation of the partition function is straightforward

$$
Z=e^{\frac{\eta}{2} \tilde{\beta}^{2} \psi_{*}^{2}-\sigma_{m} \tilde{\beta} \psi_{*}-\frac{\sigma_{m}^{2}}{2 \eta}}
$$

and the mean flow satisfies the equation

$$
\bar{q}=-\frac{1}{\tilde{\beta}} \frac{\partial \ln Z}{\partial \psi}=-\eta \tilde{\beta} \psi_{*}+\sigma_{m} .
$$

Furthermore, if the flow maximizes $S[\bar{q}]=-\frac{1}{2} \int_{D} \bar{q}^{2} d^{2} \mathbf{r}$ at fixed energy and circulation, then it is granted to be thermodynamically stable in the MRS sense [82, 39] (see also [74]). In the approach of Ellis, Haven and Turkington [37], $g(\sigma)$ is interpreted as a prior distribution for the high-order moments of potential 
vorticity (fragile constraints): arguing that real flows are subjected to forcing and dissipation at small scales, Ellis et al. [37] objected that conservation of the fragile constraints (which depend on the fine-grained field) is probably irrelevant. They suggested to treat these constraints canonically by fixing the Lagrange multiplier $\alpha_{n}$ instead of $\Gamma_{n}^{f g}$ itself. Chavanis [83, 84] showed that this is equivalent to maximizing a relative entropy $S_{\chi}=-\int \rho \ln \frac{\rho}{\chi} d^{2} \mathbf{r} d \sigma$ with a prescribed prior distribution $\chi(\sigma)$ for the small-scale potential vorticity. The ensemble-mean coarsegrained potential vorticity then is a maximum of a generalized entropy functional $S[\bar{q}]=-\int_{D} C(\bar{q}) d^{2} \mathbf{r}$ with fixed values of the robust invariants (energy, circulation and angular momentum), where $C$ is a convex function determined by the prior $\chi$ [83. The linear $q-\psi$ relation (20) corresponds to a gaussian prior $\chi$ and, in this case, the generalized entropy is minus the coarse-grained enstrophy, i.e. $S[\bar{q}]=-\frac{1}{2} \int_{D} \bar{q}^{2} d^{2} \mathbf{r}$.

As an intermediate case, Naso et al. 65] take up the argument that the conservation of some Casimirs is broken by small-scale forcing and dissipation, but instead of prescribing a prior small-scale vorticity distribution, they suggest that the relevant invariants to keep are determined directly by forcing and dissipation (which, on average, equilibrate so that the system reaches a quasi-stationary state). They show that maximizing the Miller-Robert-Sommeria entropy with fixed energy, circulation, angular momentum and fine-grained enstrophy is equivalent (for what concerns the macroscopic flow) to minimizing the coarse-grained enstrophy at fixed energy, circulation and angular momentum. Furthermore, the fluctuations around this macroscopic flow are gaussian.

Note that one does not necessarily need to justify physically the linear $q-\psi$ relationship: we may argue that we are just studying a subset of the huge and notoriously difficult to compute class of MRS statistical equilibria.

In the following sections, we shall study the mean equilibrium flow for the quasigeostrophic equations on the sphere based on these equivalent formulations of the variational problem: we use the generalized entropy $S[\bar{q}]=-\frac{1}{2}\left\langle\bar{q}^{2}\right\rangle=-\Gamma_{2}^{c g}[\bar{q}] /(2|D|)$ where $|D|=4 \pi$ is the area of the unit spher $\S$, and we consider maxima of this functional with fixed energy $E$, circulation $\Gamma$ and angular momentum $L$. Hence, our study is restricted to the case of a linear $q-\psi$ relationship. Note that from the physical point of view, it is not an irrelevant restriction as a large class of geophysical flows are described by linear $q-\psi$ relationships, like the Fofonoff flows in oceanography [85]. Besides, a strong point is that, in this limit, as we will see in the following sections, the analytical methods allow us to study a large family of metastable states that may be relevant for the atmosphere.

\subsection{Statistical ensembles and variational problems}

In this study, we shall consider the maximization of the generalized entropy $S[q]=$ $-\frac{1}{2}\left\langle q^{2}\right\rangle$, as explained in the previous section, with either fixed energy and circulation,

$\S$ In the following, the bar on $\bar{q}$ will be dropped for convenience. 
or fixed energy, circulation, and angular momentum. The corresponding variational problems can be written as

$$
\mathcal{S}(E, \Gamma)=\max _{q}\{S[q] \mid E[q]=E, \Gamma[q]=\Gamma\}
$$

and

$$
\mathcal{S}(E, \Gamma, L)=\max _{q}\{S[q] \mid E[q]=E, \Gamma[q]=\Gamma, L[q]=L\} .
$$

These constrained variational problems correspond to the microcanonical ensemble and the function $\mathcal{S}$ is called the entropy. We shall also consider the dual variational problems with relaxed constraints

$$
\mathcal{J}(\beta, \alpha)=\max _{q}\{S[q]-\beta E[q]-\alpha \Gamma[q]\},
$$

and

$$
\mathcal{J}(\beta, \alpha, \mu)=\max _{q}\{S[q]-\beta E[q]-\alpha \Gamma[q]-\mu L[q]\} .
$$

In both cases, the corresponding statistical ensemble will be termed grand-canonical $\|$. The function $\mathcal{J}$ will be called grand-potential in both cases $\mathbb{\Psi}$.

Clearly, the critical points of the microcanonical and grand-canonical variational problems are the same, due to the Lagrange multiplier theorem. However the nature of these critical points (maximum, minimum, saddle point) can differ. Nevertheless, it is straightforward to convince oneself that a maximum of the relaxed variational problem is also a maximum of the constrained variational problem (grand-canonical stability implies microcanonical stability) and that a saddle point of the constrained variational problem is necessarily a saddle point of the relaxed variational problem (microcanonical instability implies grand-canonical instability). More detailed relationships between the constrained and relaxed variational problems can be found in [36, 37, 82, 74].

It is thus possible that a maximum in the constrained variational problem (that is an equilibrium state in the microcanonical ensemble) may not be reached in the grandcanonical ensemble. Such a situation happens when no grand-canonical equilibrium has the prescribed energy, circulation, and angular momentum if relevant. In this case, we speak of ensemble inequivalence. More precisely, we have just described ensemble inequivalence at the macrostate level [36, 86]. Another characterization of ensemble inequivalence, referred to as ensemble inequivalence at the thermodynamic level, is linked to the concavity of the entropy $\mathcal{S}$ [36, 37, 86]. Indeed, the functions $\mathcal{S}$ and $\mathcal{J}$ are linked by Legendre-Fenchel transformations. Using convex analysis [87], it can be proved that the transformation is invertible only when $\mathcal{S}$ is a concave function. A particular indicator of ensemble inequivalence is the microcanonical specific heat. It is easily proved that when computed with the grand-canonical probability distribution, the specific heat is always

\| In the literature, the variational problem $\mathcal{J}(\beta, \alpha, \mu)$ is sometimes called grand-grand-canonical 74 . Here, the distinction between grand-canonical and grand-grand-canonical will always be clear because the two ensembles are considered in separate sections. Hence, we shall keep the vocabulary to a minimum and call both variational problems grand-canonical.

I The same remark as above holds for grand-potential and grand-grand-potential 
positive. On the other hand, no such result holds in the microcanonical ensemble. Thus, a negative microcanonical specific heat indicates ensemble inequivalence [88, 89, 90, 41].

In practice, the microcanonical ensemble is the natural one to treat problems where the system is large enough to be considered isolated, as in astrophysics or geophysical flows. Yet, it is always more convenient mathematically to deal with a relaxed variational problem than directly with the constrained variational problem. For this reason, it is customary to consider canonical or grand-canonical ensembles even in these cases. Meanwhile, one must keep in mind that the physical interpretation of the canonical or grand-canonical ensemble may not be straightforward. In our case, it is not easy to see what physical object would play the role of a reservoir of energy, circulation or angular momentum. In other words, it is not clear how the Lagrange multipliers $\beta, \alpha$ and $\mu$ are fixed, and which physical quantity they represent. Strictly speaking, the canonical and grand-canonical ensembles are physically relevant only when they are equivalent with the microcanonical ensemble (or when there is a good physical reason to be otherwise). On the contrary, the microcanonical ensemble is always physically relevant.

Remark: We have justified the variational principles (21)-(24) as conditions of thermodynamical stability. We note that the very same variational principles can also be interpreted as sufficient conditions of nonlinear dynamical stability with respect to the QG equations [37, 74]. Therefore, the stable states that we shall determine are both dynamically and thermodynamically stable.

\section{The equilibrium mean flow in the barotropic case $(R=\infty)$ without bottom topography $\left(h_{B}=0\right)$}

\subsection{Fixed energy and circulation}

In this section, we consider the following variational problems

$$
\mathcal{S}(E, \Gamma)=\max _{q}\{S[q] \mid E[q]=E, \Gamma[q]=\Gamma\}
$$

and

$$
\mathcal{J}(\beta, \alpha)=\max _{q}\{S[q]-\beta E[q]-\alpha \Gamma[q]\}
$$

where

$$
S[q]=-\frac{1}{2}\left\langle q^{2}\right\rangle, \quad E[q]=\frac{1}{2}\langle(q-f) \psi\rangle, \quad \Gamma[q]=\langle q\rangle,
$$

are respectively the (normalized) entropy, energy and circulation. The critical points satisfy

$$
\delta S-\beta \delta E-\alpha \delta \Gamma=0,
$$

where $\alpha$ and $\beta$ are Lagrange multipliers associated with the conservation of circulation and energy, respectively. This leads to the linear $q-\psi$ relationship

$$
q=-\beta \psi-\alpha
$$


Averaging over space, we obtain $\langle q\rangle=-\beta\langle\psi\rangle-\alpha$, but $\Gamma=\langle q\rangle=\langle-\Delta \psi+f\rangle=0$ since the integral of $\Delta \psi$ is the circulation of the velocity on the domain boundary, hence vanishing in the case of the sphere. Thus, $\alpha=-\beta\langle\psi\rangle$ and the $q-\psi$ relationship reads

$$
q=-\Delta \psi+f=-\beta(\psi-\langle\psi\rangle)
$$

If we let $\phi=\psi-\langle\psi\rangle$ such that $\langle\phi\rangle=0$, the mean field equation reduces to the simple Helmholtz equation

$$
\Delta \phi-\beta \phi=f .
$$

Here, we note that $f=2 \Omega \cos \theta=2 \Omega \sqrt{\frac{4 \pi}{3}} Y_{10}(\theta, \phi)$ is an eigenvector of the Laplacian on the sphere (with eigenvalue $\beta_{1}=-2$ ). Therefore if we introduce the operator $A_{\beta}=\Delta-\beta I, f$ satisfies $A_{\beta} f=-\left(\beta-\beta_{1}\right) f$. To solve equation (31), we have to distinguish two cases, depending whether the inverse temperature is an eigenvalue of the Laplacian or not [75].

Note that the Lagrange multiplier $\alpha$ is only related to the mean value of the stream function on the domain, $\langle\psi\rangle$, which has no effect on the structure of the flow (the stream function is defined up to an unimportant additive constant). This is not surprising as in the case considered in this section $(R=+\infty, h=f)$, we must always have $\Gamma=0$. Therefore, neither $\alpha$ nor $\Gamma$ will intervene in the following discussion. To keep the notations simple in the sequel, we shall make the gauge choice $\langle\psi\rangle=0$ and identify $\psi$ and $\phi$. This also implies $\alpha=0$.

3.1.1. Case $\beta \notin S p \Delta$ : the continuum solution In this case, equation (31) reduces to $A_{\beta}\left(\psi+\frac{f}{\beta-\beta_{1}}\right)=0$, but $A_{\beta}$ is invertible, hence

$$
\psi=-\frac{f}{\beta-\beta_{1}},
$$

and

$$
q=\frac{\beta f}{\beta-\beta_{1}} .
$$

Using equations 32 and $(33)$, and $\left\langle f^{2}\right\rangle=\frac{4}{3} \Omega^{2}$, the equilibrium energy and entropy are easily computed. We get

$$
E(\beta)=\frac{1}{2}\langle(q-f) \psi\rangle=-\beta_{1} \frac{\left\langle f^{2}\right\rangle}{2\left(\beta-\beta_{1}\right)^{2}}=\frac{4 \Omega^{2}}{3\left(\beta-\beta_{1}\right)^{2}},
$$

and

$$
S=-\frac{1}{2}\left\langle q^{2}\right\rangle=-\frac{\beta^{2}}{2\left(\beta-\beta_{1}\right)^{2}}\left\langle f^{2}\right\rangle=-\frac{2 \Omega^{2} \beta^{2}}{3\left(\beta-\beta_{1}\right)^{2}} .
$$

The relation between the energy $E$ and the Lagrange multiplier $\beta$ can be solved:

$$
\beta(E)=\beta_{1} \pm \sqrt{\frac{4 \Omega^{2}}{3 E}},
$$




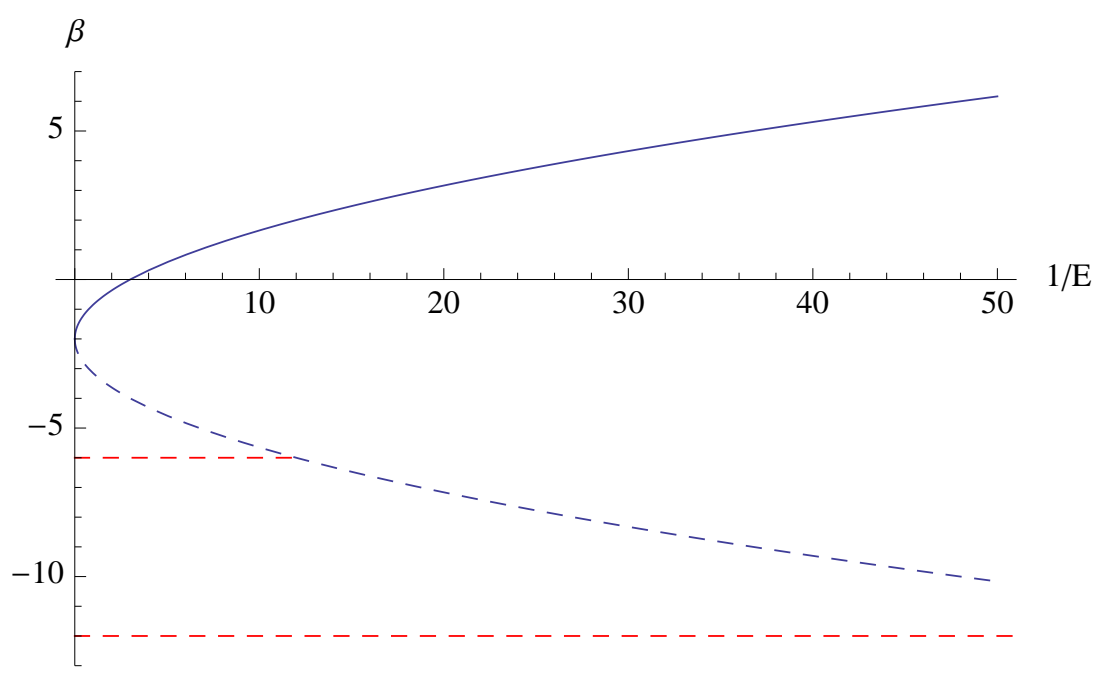

Figure 1. Caloric curve $\beta(1 / E)$ in the case where only the energy and circulation are conserved. The upper part (blue solid line) corresponds to real entropy maxima while the lower part (dashed lines) corresponds to saddle points. Horizontal lines indicate the position of the eigenvalues of the Laplacian, and therefore correspond to plateaux of (saddle) solutions.

(see the caloric curve on figure 1), so that the thermodynamic potentials $\mathcal{S}(E)$ (entropy) and $\mathcal{J}(\beta)$ (free energy) are

$$
\begin{aligned}
& \mathcal{S}(E)=-\frac{2}{3} \Omega^{2}-2 E \pm 4 \Omega \sqrt{\frac{E}{3}}, \\
& \mathcal{J}(\beta)=\frac{2}{3} \Omega^{2} \frac{\beta}{\beta_{1}-\beta} .
\end{aligned}
$$

Knowing the equilibrium streamfunction $\psi$, we can compute the zonal and meridional components of the velocity field, respectively $u$ and $v$ :

$$
u=-\frac{1}{R_{T}} \frac{\partial \psi}{\partial \theta}=-\frac{2 \Omega}{R_{T}} \frac{\sin \theta}{\beta-\beta_{1}}, \quad v=\frac{1}{R_{T} \sin \theta} \frac{\partial \psi}{\partial \phi}=0,
$$

where $R_{T}$ is the Earth's mean radius. The equilibrium motion of the fluid is thus a simple solid body rotation with angular velocity

$$
\Omega_{*}=-\frac{2 \Omega}{R_{T}^{2}\left(\beta-\beta_{1}\right)} .
$$

In particular, the equilibrium velocity distribution is purely zonal, vanishing at the poles with a maximum at the equator. At low statistical temperatures $\left(\beta<\beta_{1}=-2\right)$, the rotation of the fluid has the same sign as the solid body rotation of the Earth, while high statistical temperatures $\left(\beta>\beta_{1}=-2\right)$ correspond to counter-rotating flows. Examples of such zonal wind profiles are drawn on figure 2 for various statistical temperatures $\beta$. Note that for any given value of the energy, the two types of solutions coexist, due to the symmetry $E(-\beta-4)=E(\beta)$, as is clear from the caloric curve $\beta(E)$ shown in figure 1 .

Remark: when $\beta=0$ (corresponding to $E=\Omega^{2} / 3$ ), we find that $\Omega_{*}=-\Omega$ so that there is no rotation in the inertial frame. In a sense, the Earth rotation is canceled. 


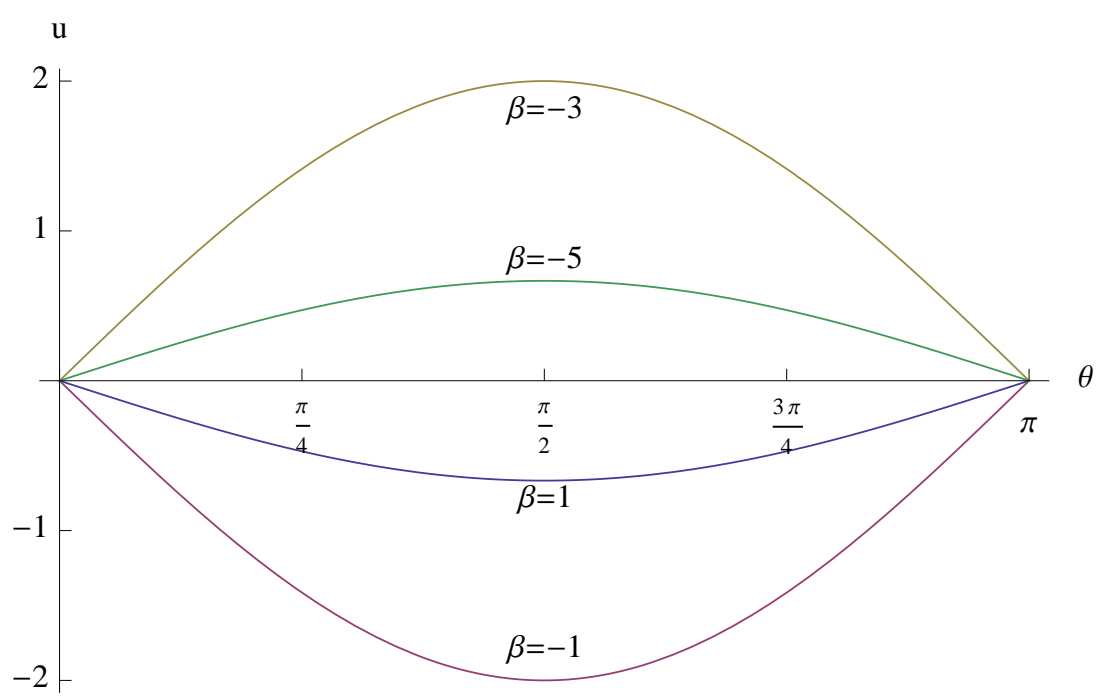

Figure 2. Zonal wind profiles for the statistical equilibrium with conservation of energy and circulation for different values of the statistical temperature. The equilibrium flow is a solid body rotation. The zonal wind is antisymmetric with respect to the transformation $\beta \rightarrow 2 \beta_{1}-\beta$. The zonal wind $u$ is normalized by the choice $R_{T}=\Omega=1$.

3.1.2. Case $\beta \in S p \Delta$ Let us now suppose that $\beta=\beta_{n}$ with $n \neq 1$. The solutions of equation (31) form a $2 n+1$ dimensional affine space: if $\psi_{0}$ is such that $A_{\beta} \psi_{0}=f$ then the space of solutions is simply $\psi_{0}+\operatorname{ker} A_{\beta}$. Specifically, the general solution reads

$$
\psi=\frac{f}{\beta_{1}-\beta_{n}}+\sum_{m=-n}^{n} \psi_{n m} Y_{n m}(\theta, \phi),
$$

where $\psi_{n m}$ are arbitrary coefficients, constrained only by the fixed value of the energy. Clearly, the expressions for the energy and the entropy become

$$
\begin{aligned}
& E=\frac{4 \Omega^{2}}{3\left(\beta_{n}-\beta_{1}\right)^{2}}-\frac{\beta_{n}}{2} \sum_{m=-n}^{n} \psi_{n m}^{2}, \\
& S=-\frac{2 \Omega^{2} \beta_{n}^{2}}{3\left(\beta_{n}-\beta_{1}\right)^{2}}-\frac{\beta_{n}^{2}}{2} \sum_{m=-n}^{n} \psi_{n m}^{2},
\end{aligned}
$$

so that the thermodynamic potentials are given by

$$
\begin{aligned}
& \mathcal{S}(E)=\beta_{n} E-\frac{2}{3} \Omega^{2} \frac{\beta_{n}}{\beta_{n}-\beta_{1}}, \\
& \mathcal{J}\left(\beta=\beta_{n}\right)=\frac{2}{3} \Omega^{2} \frac{\beta_{n}}{\beta_{1}-\beta_{n}} .
\end{aligned}
$$

For a fixed value of $\beta=\beta_{n}$, equation (42) means that the energy can have any value greater than $E\left(\beta_{n}\right)=4 \Omega^{2} /\left(3\left(\beta_{n}-\beta_{1}\right)^{2}\right)$, depending on the coefficients $\psi_{n m}$. This degeneracy is apparent in figure 1: each time the Lagrange multiplier $\beta$ reaches an eigenvalue of the Laplacian, we have a plateau of the caloric curve. The degeneracy is in fact multiple: for each point of the plateau, characterized by $\left(\beta_{n}, E_{n}\right)$, we have a whole $2 n$ dimensional sphere of solutions, with radius $\sqrt{2\left(E_{n}-E\left(\beta_{n}\right)\right) /\left(-\beta_{n}\right)}$. 
In the grand-canonical ensemble, the grand-potential has the same value for all the states on the plateau $\beta=\beta_{n}$.

Strictly speaking, $\beta=\beta_{1}$ is a forbidden value since the solution space is then empty. Nevertheless, one can consider that as $\beta \rightarrow \beta_{1}$, the streamfunction diverges proportionally to $\psi_{0}$ :

$$
\psi \sim-\frac{f}{\beta-\beta_{1}} .
$$

Similarly, the energy diverges as $\left(\beta-\beta_{1}\right)^{-2}$.

3.1.3. Nature and stability of the critical points So far, we have only found the critical points of the variational problems $(25)$ and (26). It remains to determine their nature: minimum, maximum or saddle points of the entropy functional. To that purpose, we introduce the grand-potential functional $J=S-\beta E-\alpha \Gamma$. A critical point of entropy at fixed energy and circulation is a local maximum if, and only, if

$$
\delta^{2} J=-\int_{D} \frac{(\delta q)^{2}}{2} d^{2} \mathbf{r}-\frac{\beta}{2} \int_{D}(\nabla \delta \psi)^{2} d^{2} \mathbf{r}<0,
$$

for all perturbations $\delta q$ that conserve energy and circulation at first order. This is the stability condition in the microcanonical ensemble. In the grand-canonical ensemble, the stability condition becomes $\delta^{2} J<0$ for all perturbations $\delta q$ [74].

Clearly if $\beta>0, \delta^{2} J<0$ and the point is a maximum of $S$ with respect to perturbations conserving the energy and circulation. Actually, this remains true as long as $\beta>\beta_{1}$ (see [26]). In fact, $\delta^{2} J<0$ for all perturbations $\delta q$, even those which break the conservation of the constraints: the flow is grand-canonically stable (which implies microcanonical stability). This is related to the Arnold sufficient condition of nonlinear dynamical stability [74].

Conversely, for $\beta<\beta_{1}$, let us show that the statistical equilibria computed in this section are in fact saddle points of the entropy. It suffices to consider perturbations proportional to eigenvectors of the Laplacian. Let $\delta \psi_{n m}=\epsilon Y_{n m}$ such that $\Delta \delta \psi_{n m}=$ $\beta_{n} \delta \psi_{n m}$. This perturbation conserves the circulation, and the variation of the energy at first order is $\delta E=-\epsilon \beta_{n} \int_{D} \psi Y_{n m} d^{2} \mathbf{r}$ where $\psi$ is the stream function of the basic mean flow (hence a linear combination of $Y_{00}$ and $Y_{10}$, and possibly $Y_{p m},-p \leq m \leq p, p \geq 2$ in the degenerate case). By the orthogonality property of the spherical harmonics, $\delta E=0$ if $n \geq 2(n \neq p)$ or $n=1$ and $m \neq 0$. For this particular perturbation $\delta \psi_{n m}$, we have

$$
\delta^{2} J=\beta_{n}\left(\beta-\beta_{n}\right) \int_{D} \frac{\left(\delta \psi_{n m}\right)^{2}}{2} d^{2} \mathbf{r}
$$

so that $\delta^{2} J>0$ if $\beta<\beta_{n}$. Hence, perturbations proportional to eigenvectors of the Laplacian of order $n$ with $\beta<\beta_{n}$ suffice to destabilize the flow. The particular perturbation $\delta \psi_{11}$ for instance destabilizes the equilibrium mean flow as soon as $\beta<\beta_{1}$. In particular, no degenerate mode is stable. We have proven here microcanonical instability, which implies grand-canonical instability. As a consequence, for any given energy, there is only one stable equilibrium state (solid line on figure 1 which corresponds to $\beta>-2$ ). The dashed lines on figure 1 correspond to unstable saddle points. 


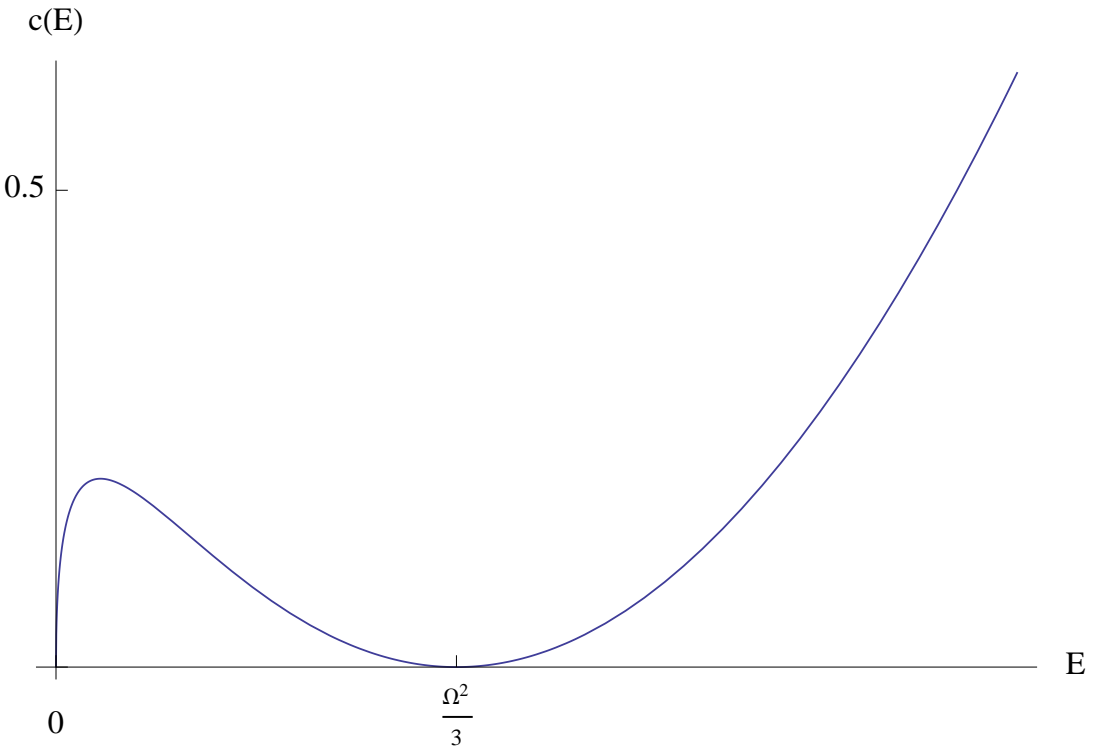

Figure 3. Heat capacity $(\partial T / \partial E)^{-1}$ in the case where only the energy and circulation are conserved. The heat capacity vanishes when $E=0$ or $E=\Omega^{2} / 3$, which is also a point of discontinuity of the microcanonical temperature $T=1 / \beta$. When $E<\Omega^{2} / 3$, the temperature is positive, otherwise it is negative.

3.1.4. Summary of the results In the microcanonical ensemble, there is only one equilibrium state (global entropy maximum) for each energy. It corresponds to the solid line on figure 1. The associated equilibrium flow is a counter-rotating solid-body rotation. The other states are unstable saddle points. In the canonical ensemble, there is an equilibrium state only for $\beta>\beta_{1}$. The other states are unstable saddle points. The ensembles are equivalent. The statistical temperature $T$ is given by

$$
\frac{1}{T}=\beta=\frac{\partial \mathcal{S}}{\partial E}=\sqrt{\frac{4 \Omega^{2}}{3 E}}-2 .
$$

It is negative when $E>\Omega^{2} / 3$. The second derivative of the entropy is negative:

$$
\frac{\partial^{2} \mathcal{S}}{\partial E^{2}}=-\frac{|\Omega|}{\sqrt{3 E^{3}}} \leq 0
$$

which means that $\mathcal{S}(E)$ is a concave function, in accordance with our findings of ensemble equivalence. Furthermore, the heat capacity $c=(\partial T / \partial E)^{-1}=$ $\left(-T^{2} \partial^{2} \mathcal{S} / \partial E^{2}\right)^{-1}$ is positive and can be computed explicitly (see figure 3 ):

$$
c=4 \sqrt{\frac{\Omega^{2}}{3}} E^{1 / 2}-8 E+4 \sqrt{\frac{3}{\Omega^{2}}} E^{3 / 2} .
$$

\subsection{Fixed energy, circulation and angular momentum}

Due to the axial symmetry, there is another relevant conserved quantity that can be taken into account in the variational problem: the angular momentum

$$
L=\langle(q-f) \cos \theta\rangle=\sqrt{\frac{4 \pi}{3}}\left\langle(q-f) \mid Y_{10}\right\rangle .
$$


The critical points of the variational problems

$$
\mathcal{S}(E, \Gamma, L)=\max _{q}\{S[q] \mid E[q]=E, \Gamma[q]=\Gamma, L[q]=L\},
$$

and

$$
\mathcal{J}(\beta, \alpha, \mu)=\max _{q}\{S[q]-\beta E[q]-\alpha \Gamma[q]-\mu L[q]\},
$$

now satisfy

$$
\delta S-\beta \delta E-\alpha \delta \Gamma-\mu \delta L=0,
$$

which leads to the $q-\psi$ relationship $q=-\beta \psi-\alpha-\mu \cos \theta$. According to section 2.2, the solutions of this equation correspond to states that are steady in a frame rotating with angular velocity $\Omega_{L}=\mu / \beta$ : indeed, this relation is of the form $q=F\left(\psi+\frac{\mu}{\beta} \cos \theta\right)$ with $F(x)=-\beta x-\alpha$. Imposing $\mu=0$, that is neglecting conservation of the angular momentum, amounts to considering only the solutions which are stationary in the reference frame rotating with the angular velocity of the Earth. These solutions were described in the previous section. As in the previous section, spatial averaging yields $\alpha=-\beta\langle\psi\rangle$ and the $q-\psi$ relationship becomes

$$
q=-\beta(\psi-\langle\psi\rangle)-\mu \cos \theta .
$$

Making again the gauge choice $\langle\psi\rangle=0$, leading to $\alpha=0$, and setting $\tilde{f}=f+\mu \cos \theta=$ $(2 \Omega+\mu) \cos \theta$, we find that $\psi$ is given by the Helmholtz equation

$$
A_{\beta} \psi=\tilde{f} .
$$

We now discuss the resolution of the Helmholtz equation (57) as in the previous section.

3.2.1. Case $\beta \notin S p \Delta$ : the continuum solution In this case, $A_{\beta}$ is invertible and $\psi$ is proportional to the first eigenmode of the Laplacian, so that

$$
\psi=-\frac{\tilde{f}}{\beta-\beta_{1}}=\frac{2 \Omega+\mu}{\beta_{1}-\beta} \cos \theta .
$$

The equilibrium flow is a solid-body rotation with angular velocity

$$
\Omega_{*}=\frac{2 \Omega+\mu}{\beta_{1}-\beta} .
$$

The potential vorticity is $q=2\left(\Omega+\Omega_{*}\right) \cos \theta$. We can compute the energy, angular momentum, and entropy:

$$
\begin{aligned}
& E=-\frac{\beta_{1}}{2} \Omega_{*}{ }^{2}\left\langle\cos ^{2} \theta\right\rangle=\frac{1}{3}\left(\frac{2 \Omega+\mu}{\beta-\beta_{1}}\right)^{2}, \\
& L=\frac{2}{3} \Omega_{*}=\frac{2}{3} \frac{2 \Omega+\mu}{\beta_{1}-\beta}, \\
& S=-\frac{2}{3}\left(\Omega+\Omega_{*}\right)^{2}=-\frac{2}{3}\left(\frac{\mu-\beta \Omega}{\beta_{1}-\beta}\right)^{2} .
\end{aligned}
$$


The thermodynamic potentials $\mathcal{S}(E, L)$ and $\mathcal{J}(\beta, \mu)$ are given by

$$
\begin{aligned}
& \mathcal{S}(E, L)=-\frac{2}{3} \Omega^{2}-2 \Omega L-2 E=-\frac{3}{2}\left(L+\frac{2}{3} \Omega\right)^{2} \\
& \mathcal{J}(\beta, \mu)=\frac{1}{3} \frac{(2 \Omega+\mu)^{2}}{\beta-\beta_{1}}-\frac{2}{3} \Omega^{2} .
\end{aligned}
$$

As always true for solid-body rotations (see Appendix B), the energy and angular momentum are linked by $E=E^{*}(L)$, with $E^{*}(L)=3 L^{2} / 4$. This relation is independent of $\beta$. Hence in the microcanonical ensemble, the continuum solution exists only on the curve $E=E^{*}(L)$. For $E>E^{*}(L)$ there is no such solution.

3.2.2. Case $\beta \in S p \Delta$ Let us suppose that $\beta=\beta_{n}$ with $n \neq 1$. As in section 3.1.2, the general solution of the Helmholtz equation (57) when $\beta$ is an eigenvalue of the Laplacian is a superposition of eigenmodes given by

$$
\begin{aligned}
\psi & =-\frac{\tilde{f}}{\beta_{n}-\beta_{1}}+\sum_{m=-n}^{n} \psi_{n m} Y_{n m}(\theta, \phi) \\
& =\Omega_{n}^{*} \cos \theta+\sum_{m=-n}^{n} \psi_{n m} Y_{n m}(\theta, \phi),
\end{aligned}
$$

where $\Omega_{n}^{*}=\frac{2 \Omega+\mu}{\beta_{1}-\beta_{n}}$ and $\psi_{n m}$ are arbitrary coefficients. The requirement for the stream function to be real-valued imposes $\psi_{n,-m}=\psi_{n m}^{*}$. The corresponding energy, angular momentum and entropy are given by

$$
\begin{aligned}
& E=\frac{\Omega_{n}^{* 2}}{3}-\frac{\beta_{n}}{2} \sum_{m=-n}^{n}\left|\psi_{n m}\right|^{2} \\
& L=\frac{2}{3} \Omega_{n}^{*} \\
& S=-\frac{2}{3}\left(\Omega+\Omega_{n}^{*}\right)^{2}-\frac{\beta_{n}^{2}}{2} \sum_{m=-n}^{n}\left|\psi_{n m}\right|^{2} .
\end{aligned}
$$

The Lagrange multiplier $\mu$, associated with the conservation of angular momentum, is determined by the relation $L=2 \Omega_{n}^{*} / 3$ which can be inverted to yield

$$
\mu=\frac{3}{2}\left(\beta_{1}-\beta_{n}\right) L-2 \Omega .
$$

The entropy $\mathcal{S}(E, L)$ and grand-potential $\mathcal{J}(\beta, \mu)$ are given by

$$
\begin{aligned}
& \mathcal{S}(E, L)=\beta_{n}\left(E-E^{*}(L)\right)-\frac{3}{2}\left(L+\frac{2}{3} \Omega\right)^{2}, \\
& \mathcal{J}\left(\beta=\beta_{n}, \mu\right)=\frac{1}{3} \frac{(2 \Omega+\mu)^{2}}{\beta_{n}-\beta_{1}}-\frac{2}{3} \Omega^{2} .
\end{aligned}
$$

We shall see that these solutions are unstable saddle points in both ensembles. In the microcanonical ensemble, when $E=E^{*}(L)$, this degenerate solution reduces to the continuum solution. 
3.2.3. Case $\beta=\beta_{1}$ In this case, equation (57) admits solutions only if the right-hand side vanishes, i.e. when $\mu=\mu_{c} \equiv-2 \Omega$. Then, the equilibrium flow has the general form

$$
\psi=\psi_{10} Y_{10}(\theta, \phi)+\psi_{11} Y_{11}(\theta, \phi)+\psi_{11}^{*} Y_{1,-1}(\theta, \phi),
$$

where $\psi_{10}$ is a real coefficient and $\psi_{11}$ a complex coefficient, linked by the energy and angular momentum requirements. Setting $\Omega_{*}=\sqrt{\frac{3}{4 \pi}} \psi_{10}, \gamma_{c}=\sqrt{\frac{3}{2 \pi}} \Re \psi_{11}, \gamma_{s}=$ $-\sqrt{\frac{3}{2 \pi}} \Im \psi_{11}$, the energy, angular momentum and entropy read

$$
\begin{aligned}
& E=\frac{1}{3}\left(\Omega_{*}^{2}+\gamma_{c}^{2}+\gamma_{s}^{2}\right), \\
& L=\frac{2}{3} \Omega_{*}, \\
& S=-\frac{2}{3}\left(\left(\Omega+\Omega_{*}\right)^{2}+\gamma_{c}^{2}+\gamma_{s}^{2}\right),
\end{aligned}
$$

so that $\Omega_{*}$ is in fact fixed by the angular momentum $L$ while $\gamma_{c}$ and $\gamma_{s}$ depend on both $E$ and $L$ :

$$
\begin{aligned}
& \Omega_{*}=\frac{3}{2} L, \\
& \gamma_{c}^{2}+\gamma_{s}^{2}=3\left(E-E^{*}(L)\right) .
\end{aligned}
$$

Introducing the angle $\phi_{0}$ such that $\gamma_{c}=\sqrt{3\left(E-E^{*}(L)\right)} \cos \phi_{0}$ and $\gamma_{s}=$ $\sqrt{3\left(E-E^{*}(L)\right)} \sin \phi_{0}$, the stream function reads

$$
\begin{aligned}
\psi & =\Omega_{*} \cos \theta+\gamma_{c} \sin \theta \cos \phi+\gamma_{s} \sin \theta \sin \phi \\
& =\Omega_{*} \cos \theta+\sqrt{3\left(E-E^{*}(L)\right)} \sin \theta \cos \left(\phi-\phi_{0}\right) .
\end{aligned}
$$

When $E=E^{*}(L)$, this solution coincides with the continuum solution: it is a solid-body rotation. When $E>E^{*}(L)$, the flow has wave-number one in the longitudinal direction; it is a dipole with the angle $\phi_{0}$ playing the role of a phase. The phase $\phi_{0}$ is arbitrary (it is not determined by the constraints). The stream function can be re-written as

$$
\psi=\frac{3}{2} L\left[\cos \theta+\sqrt{\frac{E}{E^{*}(L)}-1} \sin \theta \cos \left(\phi-\phi_{0}\right)\right] .
$$

Therefore, the amplitude of the dipole depends on a single control parameter $\epsilon \equiv$ $E / E^{*}(L)$ and is given by $a(\epsilon)=(\epsilon-1)^{1 / 2}$ (on the other hand $\frac{3}{2} L$ just fixes the amplitude). If we interpret $a$ as the order parameter, this corresponds to a second order phase transition occurring for $\epsilon \geq \epsilon_{c}=1$ between a "solid rotation" phase and a "dipole" phase (see figure 4). Sample stream functions are shown in figure 4 for various values of $\epsilon$. The position of the dipole depends on the value of $\epsilon$ : the larger $\epsilon$, the more the dipole is aligned along the equator.

Note also that the thermodynamic potentials can be expressed as

$$
\begin{aligned}
& \mathcal{S}(E, L)=-2\left(E-E^{*}(L)\right)-\frac{3}{2}\left(L+\frac{2}{3} \Omega\right)^{2}, \\
& \mathcal{J}\left(\beta=\beta_{1}, \mu=\mu_{c}\right)=-\frac{2}{3} \Omega^{2} .
\end{aligned}
$$




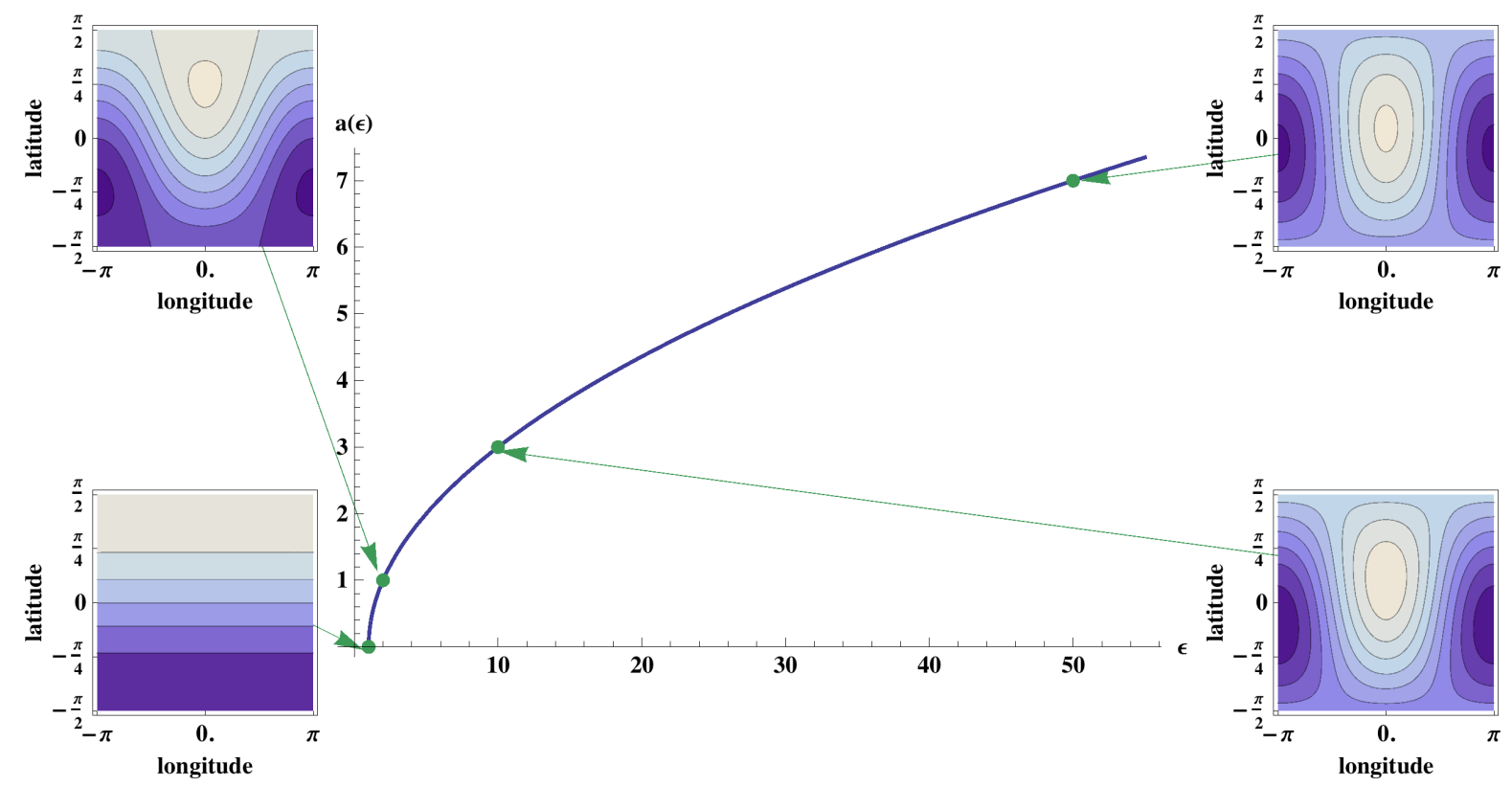

Figure 4. Amplitude of the dipole as a function of the control parameter $\epsilon \equiv E / E^{*}(L)$. There is a second order phase transition at $\epsilon_{c}=1$ between a "solid-body rotation" phase $\left(\epsilon=\epsilon_{c}\right)$ and a "dipole" phase $\left(\epsilon>\epsilon_{c}\right)$. Insets show particular stream functions for specific values of $\epsilon$. Here $\phi_{0}=0$.

These relations have two implications: (i) the solutions with different $\phi_{0}$ have the same entropy (which was expected) so they are statistically equivalent, and (ii) these solutions have a higher entropy than the solutions with $\beta=\beta_{n>1}$. As a consequence of (i), the second order phase transition is accompanied by spontaneous symmetry breaking, as the phase of the dipole is arbitrary.

Remark: The condition $\mu=-2 \Omega$ with $\beta=\beta_{1}=-2$ corresponds to $\Omega_{L}=\mu / \beta=\Omega$. Therefore, the dipole is stationary in a frame rotating with angular velocity $\Omega$ with respect to the Earth (hence, rotating at the angular velocity $2 \Omega$ with respect to the inertial frame).

3.2.4. Nature and stability of the critical points A critical point of entropy at fixed energy, circulation and angular momentum is a local maximum if, and only, if

$$
\delta^{2} J=\delta^{2} S-\beta \delta^{2} E=-\int_{D} \frac{(\delta q)^{2}}{2} d^{2} \mathbf{r}-\frac{\beta}{2} \int_{D}(\nabla \delta \psi)^{2} d^{2} \mathbf{r}<0,
$$

for all perturbations $\delta q$ that conserve energy, circulation and angular momentum at first order. We have introduced the grand-potential functional $J[q]=S[q]-\beta E[q]-$ $\alpha \Gamma[q]-\mu L[q]$. This is the stability condition in the microcanonical ensemble. In the grand-canonical ensemble, the stability condition becomes $\delta^{2} J<0$ for all perturbations $\delta q[74]$.

Carrying out the same analysis as in section 3.1.3, one concludes that the critical points found previously are entropy maxima only if $\beta>\beta_{1}$. As in section 3.1.3, if $\beta>\beta_{1}$, the flow is grand-canonically stable (i.e. stable for all perturbations $\delta q$ and not 
only those which preserve the constraints at first order) and thus also microcanonically stable.

Otherwise, one can exhibit perturbations that destabilize the mean flow. Indeed, at first order, perturbations of the type $\delta \psi_{n m}=\epsilon Y_{n m}$ conserve the circulation as previously. Since $\delta L=\left\langle\cos \theta \delta q_{n m}\right\rangle$ and $\cos \theta$ is proportional to $Y_{10}, \delta \psi_{n m}$ conserves the angular momentum if $(n, m) \neq(1,0)$. Besides, as $\delta E=-\beta_{n}\left\langle\psi \delta \psi_{n m}\right\rangle$, the perturbation conserves energy for $(n, m) \neq(0,0),(1,0)$ in the case of the continuum solution, and for $(n, m) \neq(0,0),(1,0), n \neq p$ when $\beta=\beta_{p}$. Furthermore, since $\delta^{2} J=\beta_{n}\left(\beta-\beta_{n}\right) \int_{D} \frac{\left(\delta \psi_{n m}\right)^{2}}{2} d^{2} \mathbf{r}$, these perturbations destabilize the mean flow if, and only, if $\beta<\beta_{n}$. In particular, as soon as $\beta<\beta_{1}$, the mean flow is not stable with respect to the perturbation $\delta \psi_{11}$ for instance. All the equilibrium flows with $\beta<\beta_{1}$ are thus saddle points. Again, as in section 3.1.3, we have proved microcanonical instability, which implies grand-canonical instability.

It remains to be seen what happens when $\beta=\beta_{1}$. In that case, the quadratic form $\delta^{2} J$ is degenerate. The vector space spanned by $Y_{11}, Y_{1,-1}$ and $Y_{10}$ constitutes the radical of $\delta^{2} J$ : the function $J$ is constant on this vector space (with value $-2 \Omega^{2} / 3$ ). Hence we have a three-dimensional vector space of metastable states in the grandcanonical ensemble. Spontaneous perturbations may be generated at no cost in inverse temperature $\beta$ and Lagrange multiplier $\mu$, which induce transitions between one dipole to another, possibly with different values of energy, angular momentum, and phase. In the microcanonical ensemble, as we fix the values of the energy and angular momentum, the only such perturbation which is possible is that which changes the phase of the dipole. Hence we only have a one-dimensional manifold of metastable states in the microcanonical ensemble. These spontaneous perturbations can be interpreted in terms of Goldstone bosons, as they appear due to continuous symmetry breaking [91].

3.2.5. Summary of the results To sum up the results obtained in the previous sections, we start by treating the grand-canonical ensemble where $\beta$ and $\mu$ are fixed:

- If $\mu \neq \mu_{c}$, the only stable equilibrium state is a solid-body rotation $\Omega_{*}<0$, obtained for $\beta>\beta_{1}$. Two types of saddle points are possible for $\beta<\beta_{1}$ : solid-body rotation $\Omega_{*}>0$ when $\beta$ is not an eigenvalue of the Laplacian, or more structured flows when $\beta$ is an eigenvalue of the Laplacian but these solutions are unstable. Finally, there is no solution with $\beta=\beta_{1}$.

- If $\mu=\mu_{c}$, the continuum solution is the trivial motionless solution: $\Omega_{*}=0$ (and thus $E=0, L=0$ for all values of $\beta$, see figure 5). The eigenmodes solutions remain accessible but unstable, while a new dipole solution appears for $\beta=\beta_{1}$, with arbitrary energy and angular momentum (see figures 5 and 6). This corresponds to a second order phase transition with spontaneous symmetry-breaking.

For both cases, the caloric curve $E(\beta)$ is shown, for different values of $\mu$, in figure 5. Similarly, the curve $L(\mu)$ is shown on figure 6 for different values of $\beta$ : for $\beta \neq \beta_{1}$, it is a straight line with slope $2 /\left(3\left(\beta_{1}-\beta\right)\right)$. For $\beta=\beta_{1}$, it is a vertical line at $\mu=-2 \Omega$ 

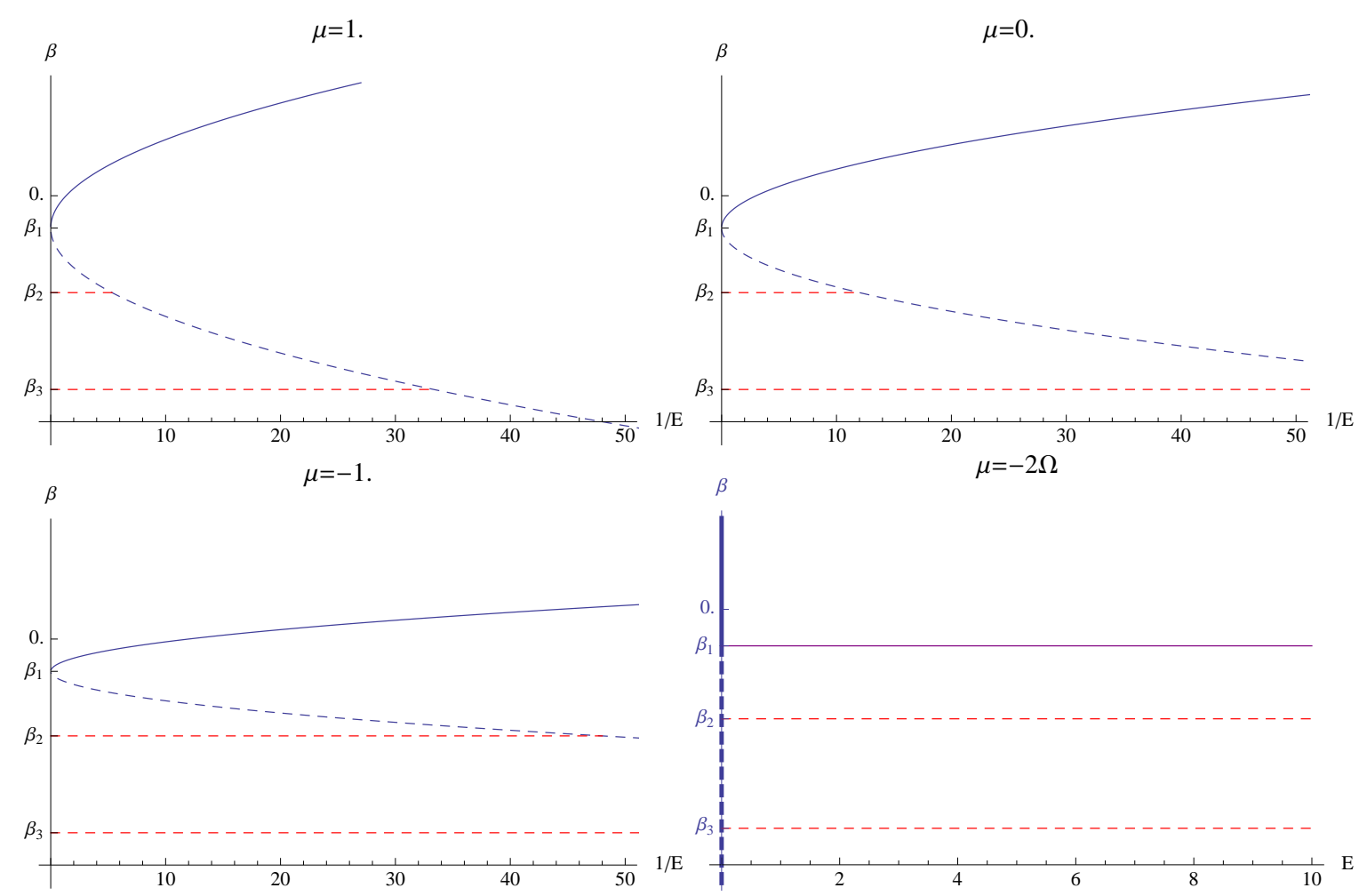

Figure 5. Caloric curves $1 / E(\beta)$ (respectively $E(\beta)$ for the lower-right panel) for different values of the Lagrange parameter $\mu$ in the grand-canonical ensemble. From left to right and from top to bottom, $\mu=1,0,-1$ and $\mu=-2 \Omega$. The solid blue line (continuum solution, solid-body rotation) corresponds to true maxima of the grand-potential while the dashed blue line corresponds to saddle-points (still for the continuum solution). Dashed horizontal red lines indicate the position of the eigenvalues of the Laplacian, and therefore correspond to plateaux of degenerate (saddle) solutions. In the lower-right panel, $\mu+2 \Omega=0$ : the continuum solution only exists on the axis $E=0$. The solid purple line represents the symmetry-breaking dipole solution.

indicating that the value of the angular momentum is arbitrary. These results are summarized in the grand-canonical phase diagram (figure 7).

Now, in the microcanonical ensemble, the equilibrium is determined by the given value of $(E, L)$ as follows:

- If $E=E^{*}(L)$, the stable equilibrium is a solid-body rotation with angular velocity $\Omega_{*}=3 L / 2$. Note that in this case, the Lagrange multipliers $\beta$ and $\mu$ are not determined by $E$ and $L$ (see figures 8 and 9 ). The only constraints are $\beta>\beta_{1}$ and $\mu<\mu_{c}$ or $\mu>\mu_{c}$ depending on the sign of $L$. In other words, for $E=E^{*}(L)$, the caloric curve $\beta(E)$ (figure 8) and the chemical potential line $\mu(L)$ (figure 9) are vertical lines.

- If $E>E^{*}(L)$, the most probable state is the dipole of section 3.2.3, with an undetermined phase $\phi_{0}$. This is a case of spontaneous symmetry breaking, insofar as the longitude dependence of one particular solution (for a given $\phi_{0}$ ) breaks the 
L

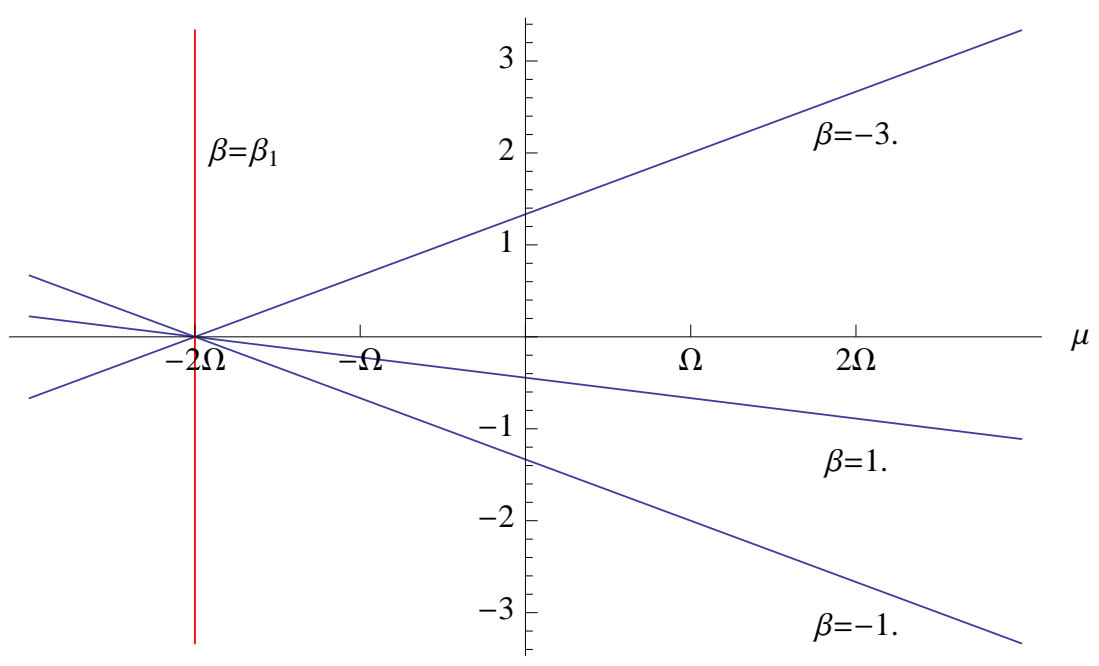

Figure 6. Chemical potential curve $L(\mu)$ for different values of the temperature $\beta$ in the grand-canonical ensemble. For every value of $\beta$, the curve is a straight line. For all $\beta \neq \beta_{1}$, it has a finite slope $2 /\left(3\left(\beta_{1}-\beta\right)\right)$. When $\beta=\beta_{1}$, necessarily $\mu=-2 \Omega$, and the value of the angular momentum does not depend on $\mu$.

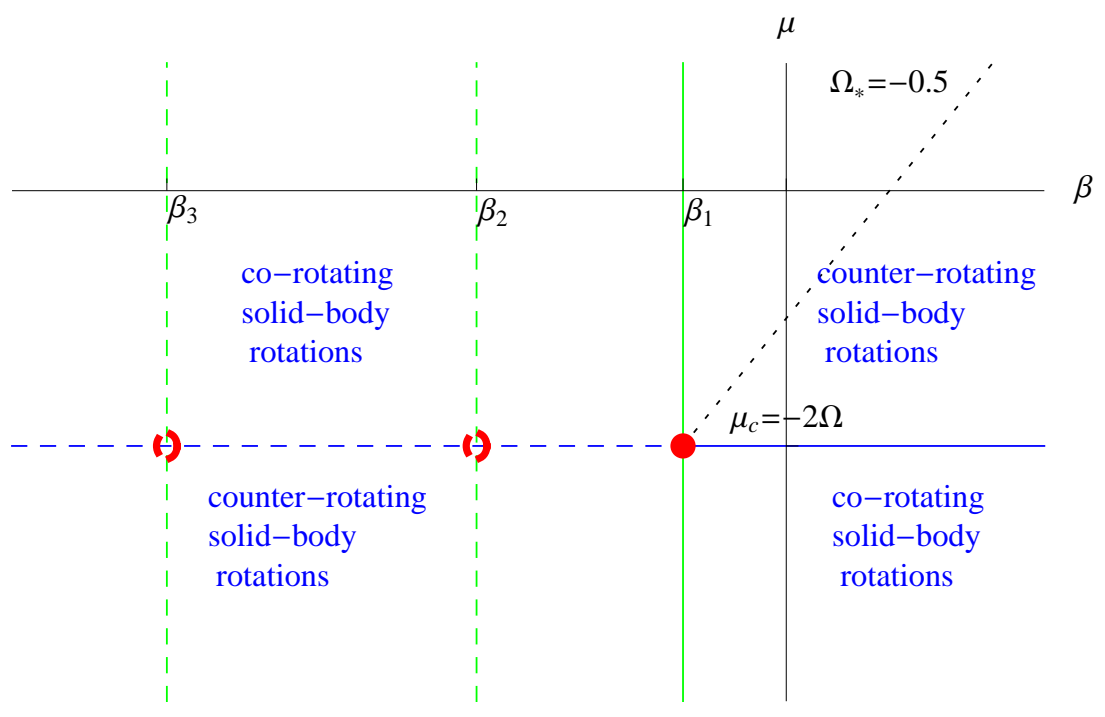

Figure 7. Phase diagram in the grand-canonical ensemble. When $\beta>\beta_{1}$, the equilibrium state is a solid-body rotation, co-rotating or counter-rotating depending on the position of $\mu$ with respect to $-2 \Omega$. The solid blue line indicates the separating case of a motionless flow. When $\beta=\beta_{1}$ and $\mu=-2 \Omega$, the equilibrium flow is a symmetrybreaking dipole. There is a second order phase transition at this point (red dot). When $\beta=\beta_{n}, n \neq 1$ (dashed green lines), solid-body rotations coexist with degenerate states, but they are all unstable. Only the degenerate states remain when $\mu=-2 \Omega$ (dashed red circles). The dashed blue line corresponds to an unstable motionless case, while the solid green line is an impossible case (no solution to the mean-field equation). The dotted half straight line represents an iso- $\Omega_{*}$ line (corresponding to $\Omega_{*}=-0.5$ ). 


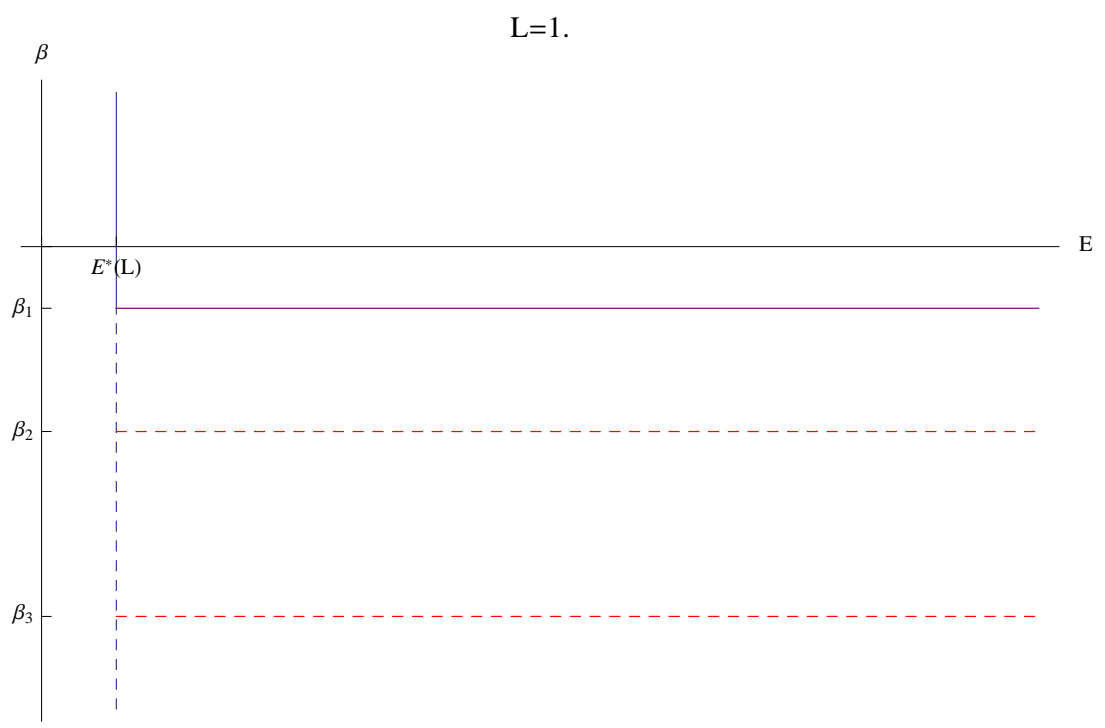

Figure 8. Caloric curve $\beta(E)$ in the microcanonical ensemble, in the case when the energy, circulation and angular momentum are conserved. For a given value of the angular momentum $L$ (here $L=1$ ), the energy is necessarily greater than $E^{*}(L)$. When $E>E^{*}(L)$, the only stable equilibrium is obtained for $\beta=\beta_{1}$ (solid purple line, dipole). However, there are an infinity of saddle points corresponding to $\beta=\beta_{n}$ (dashed red lines, degenerate states). When $E=E^{*}(L), \beta$ is not fixed and can take any value. In this case, the flow is a solid-body rotation. Cases $\beta>\beta_{1}$ correspond to stable equilibria while $\beta<\beta_{1}$ correspond to saddle points. Note that the value of the angular momentum $L$ only modifies the position of the point $E^{*}(L)$, but does not change the shape of the microcanonical caloric curve.

axial symmetry. However, as usual, the ensemble of solutions satisfy the axial symmetry. Furthermore, there are degenerate solutions which are unstable saddle points with a lower entropy. The caloric curve $\beta(E)$ at fixed angular momentum (figure 8 consists of an ensemble of horizontal lines. The horizontal line with $\beta=\beta_{1}$ corresponds to the equilibrium dipole flow: the statistical temperature does not depend on the energy. In addition, there are horizontal lines at $\beta=\beta_{n}, n>1$ corresponding to the unstable degenerate states. Similarly, for fixed energy $E$, the chemical potential curve $\mu(L)$ (figure 9) is a horizontal line at $\mu=\mu_{c}$ for the (stable) dipole equilibrium. There are also an infinity of straight lines corresponding to degenerate modes with $\beta=\beta_{n}, n>1$, with slopes $3\left(\beta_{1}-\beta_{n}\right) / 2$, but these modes are unstable saddle points.

Recall that a flow with energy $E$ and angular momentum $L$ necessarily satisfies $E \geq E^{*}(L)$ (see Appendix B). Therefore our classification of the final equilibrium state reached by the flow is complete; it is summarized in the microcanonical phase diagram of figure 10 . The line $E=E^{*}(L)$ corresponds to a line of second order phase transition with spontaneous symmetry breaking: on this line, the equilibrium is a solid-body rotation (with a direction given by the sign of the angular momentum); above the line, the equilibrium is a dipole flow with amplitude $a(\epsilon)$ and an arbitrary phase. 


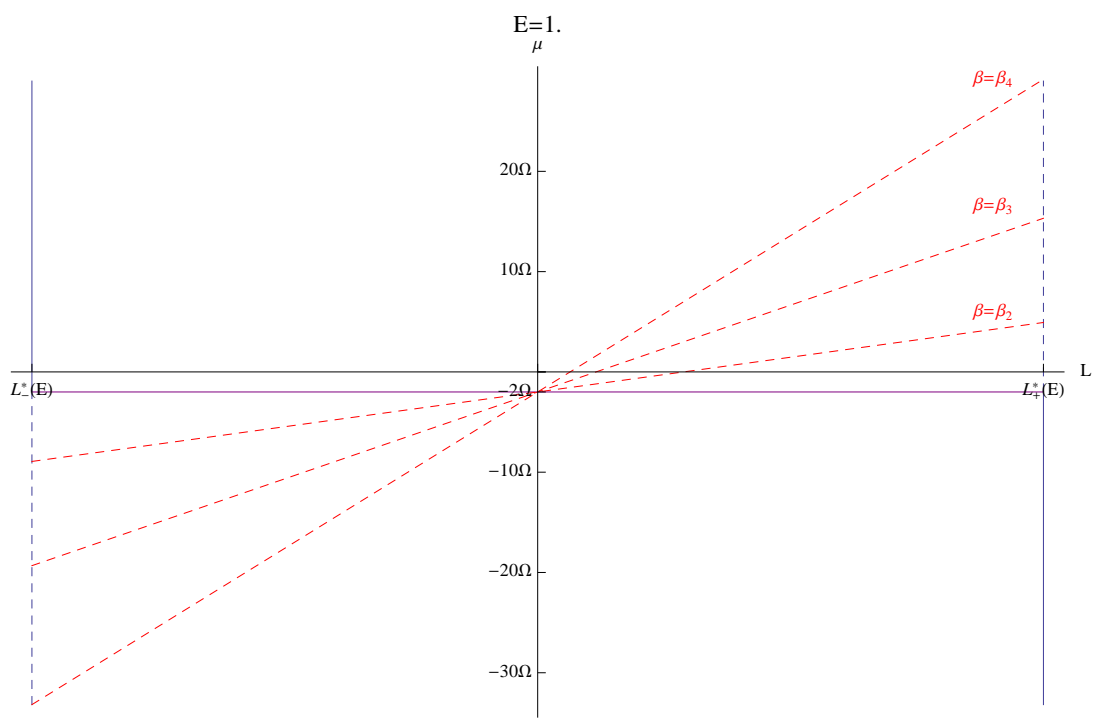

Figure 9. Chemical potential $\mu(L)$ in the microcanonical ensemble, in the case of conservation of energy, circulation and angular momentum. For a given value of $E$ (here $E=1), L$ lies between $L_{-}^{*}(E)$ and $L_{+}^{*}(E)$. The two straight lines $L=L_{-}^{*}(E)$ and $L=L_{+}^{*}(E)$ correspond to solid-body rotations. In this case, the value of the parameter $\mu$ is not fixed by $L$ as only the angular velocity $\Omega_{*}$, which is a function of both $\mu$ and $\beta$, is important. The solid blue line represents stable solid-body rotations while the dashed blue line corresponds to unstable solid-body rotations. The straight line $\mu=-2 \Omega$ (solid purple) corresponds to the case of the dipole flow, which occurs when $|L| \neq L_{+}^{*}(E)$. There are an infinity of straight lines with slopes $3\left(\beta_{1}-\beta_{n}\right) / 2$ (three of them are represented with dashed red on the figure for $n=2,3,4)$, corresponding to unstable degenerate states.

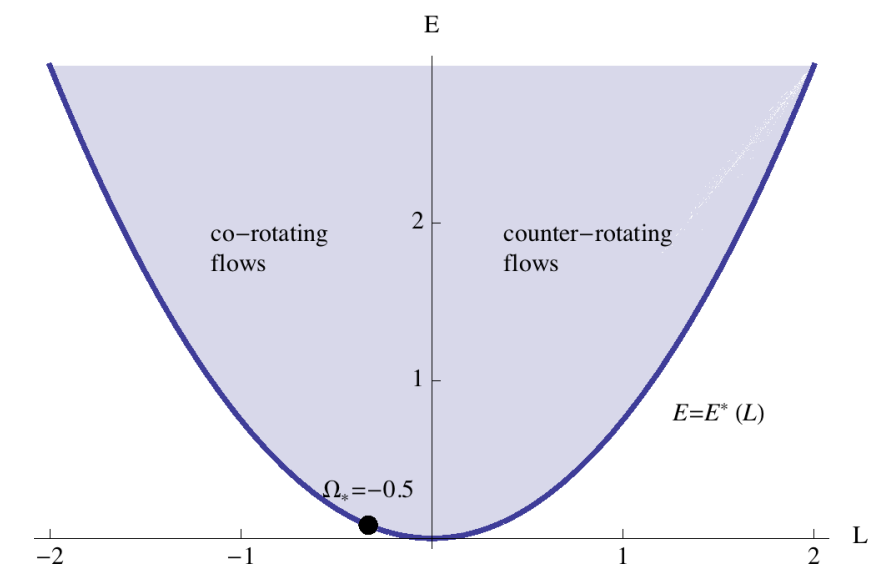

Figure 10. Phase diagram in the microcanonical ensemble: the final state of the flow predicted by statistical mechanics depends on the position in the $(E, L)$ space. The thick blue line represents the curve $E=E^{*}(L)$ defined in the text. On this curve, the statistical equilibrium is a solid-body rotation (counter-rotating for $L>0$ and co-rotating for $L<0$ ) with angular velocity $\Omega_{*}$ (we have shown $\Omega_{*}=-0.5$ ). In the portion of the plane lying over this curve (blue filled area), the statistical equilibrium is the dipolar flow of section 3.2.3. The blue parabola is the locus of a second order phase transition with spontaneous symmetry breaking. The portion under the curve is forbidden by the energy inequality obtained in Appendix B. 
3.2.6. Discussion of the ensemble equivalence properties Contrary to other studies with the same model (quasi-geostrophic equations) but in a different geometry [39, 40], the microcanonical and the grand-canonical ensemble are equivalent here. However, the ensemble equivalence is only partial in the standard terminology [36]: we have seen that at the macrostates level, the equilibrium states reached in the grand-canonical ensemble and in the microcanonical ensemble are the same. More precisely, each set of equilibrium states obtained in the microcanonical ensemble (at fixed $(E, L)$ with $\left.E>E^{*}(L)\right)$ is a proper subset of the set of grand-canonical states obtained at the corresponding Lagrange multiplier $\left(\beta=\beta_{1}, \mu=\mu_{c}\right)$. This is the general case of partial ensemble equivalence. Here the situation is rather extreme as the set of grand-canonical equilibrium states obtained for a single value of the $(\beta, \mu)$ couple contains all the microcanonical equilibrium states for any value of the energy and angular momentum. In other words, the point $\left(\beta=\beta_{1}, \mu=\mu_{c}\right)$ in the grand-canonical phase diagram is mapped onto the whole domain $E>E^{*}(L)$ in the microcanonical phase diagram. As far as solid-body rotations are concerned, each half straight line corresponding to a fixed angular velocity in the grand-canonical phase diagram is mapped onto a point on the $E=E^{*}(L)$ parabola in the microcanonical phase diagram (see figures 7 and 10 ). Partial ensemble equivalence is also seen at the thermodynamic level: geometrically, the entropy $\mathcal{S}(E, L)=-\frac{2}{3} \Omega^{2}-2 \Omega L-2 E$ is a plane. In particular, it is a concave function, but only marginally so; it is also a convex function. The statistical temperature $1 / T=\beta=\partial \mathcal{S} / \partial E$ is constant and equal to $\beta_{1}$, except possibly when $E=E^{*}(L)$. Besides, both second partial derivatives $\partial^{2} \mathcal{S} / \partial E^{2}$ and $\partial^{2} \mathcal{S} / \partial L^{2}$ vanish.

Here, it is possible to measure how severe the partial ensemble equivalence is. We have already described precisely the relationships between the different sets of equilibrium states obtained for various values of the parameters in both statistical ensembles. Now, we recall that in the stability analysis, we mentioned that in the grandcanonical ensemble, there is metastability in a three-dimensional vector space, while it reduces to a one-dimensional manifold in the microcanonical ensemble. In other words, there are three different modes (Goldstone modes) which can move the system from one metastable state to another in the grand-canonical ensemble, while there is only one such Goldstone mode in the microcanonical ensemble. If we considered any mixed ensemble, with one constraint treated microcanonically and the other canonically, we would have two Goldstone modes. Thus, the number of Goldstone modes in each ensemble provides a refined characterization of ensemble equivalence properties, as compared to simply calling it "partial".

The phase transition observed here is made possible by the degeneracy of the first eigenspace of the Laplacian on the sphere, which allows for non-trivial energy condensation. Although all the energy condenses in the first mode, we have two distinct equilibrium flow structures. The ensemble equivalence properties also owe to the particular geometry. Previous studies all assumed that the first eigenvalue of the Laplacian is non-degenerate [39, 40, 92, 93]. When this is not the case, it is straightforward to see that ensemble inequivalence results such as those obtained in 
[39] may collapse.

Nonetheless, it is not clear how generic partial equivalence of statistical ensemble is. For instance, considering small non-linearities in the $q-\psi$ relationship may change the nature of the phase transition and the ensemble equivalence properties. In the case of the energy-enstrophy ensemble on a rectangular domain with fixed boundary conditions, it has been shown [93] that the phase transition may remain second order or turn into a first-order phase transition depending on the sign of the first non-linear coefficient in the $q-\psi$ relationship. This analysis is likely to remain valid in the case we are considering here.

\section{General case: quasi-geostrophic flow over a topography.}

In the previous section, we have seen that in the absence of a bottom topography, the statistical mechanics of the quasi-geostrophic equations in spherical geometry can be solved in a very simple manner due to the fact that the Coriolis parameter is an eigenvector of the Laplacian on the sphere. Adding an angular momentum conservation constraint does not alter this derivation since it simply adds another contribution proportional to the same eigenvector of the Laplacian to the mean field equation. In this section, we treat the general case, with a finite Rossby deformation radius as well as an arbitrary topography.

\subsection{The general mean field equation and its solution}

The critical points of the variational problem

$$
\mathcal{S}(E, \Gamma, L)=\max _{q}\{S[q] \mid E[q]=E, \Gamma[q]=\Gamma, L[q]=L\},
$$

given by $\delta S-\beta \delta E-\alpha \delta \Gamma-\mu \delta L=0$, satisfy the linear $q-\psi$ relationship $q=$ $-\beta \psi-\alpha-\mu \cos \theta$. Now, $\alpha$ is determined by averaging over the whole domain

$$
\Gamma=\langle q\rangle=\frac{\langle\psi\rangle}{R^{2}}+\langle h\rangle=-\beta\langle\psi\rangle-\alpha .
$$

Replacing $q$ with $-\Delta \psi+\frac{\psi}{R^{2}}+h$, the mean field equation becomes

$$
A_{\lambda}(\psi-\langle\psi\rangle)=h-\langle h\rangle+\mu \cos \theta
$$

where $\lambda=\beta+\frac{1}{R^{2}}$ plays the role of the inverse temperature $\beta$ in the case of a finite Rossby deformation radius. As before, we are free to make the gauge choice $\langle\psi\rangle=0$ as it does not affect the structure of the flow. Therefore, the mean field equation can be rewritten

$$
-\Delta \psi+\lambda \psi=\langle h\rangle-h-\mu \cos \theta .
$$

Following [63], the general solution can be written

$$
\psi=\phi_{1}+\mu \phi_{2}
$$


where $\phi_{1}$ and $\phi_{2}$ satisfy

$$
\begin{aligned}
-\Delta \phi_{1}+\lambda \phi_{1} & =\langle h\rangle-h, \\
-\Delta \phi_{2}+\lambda \phi_{2} & =-\cos \theta
\end{aligned}
$$

Let us assume for the moment that $\lambda \notin \operatorname{Sp} \Delta$. We have

$$
\phi_{1}=-\sum_{n \neq 0} \sum_{m=-n}^{n} \frac{\left\langle h \mid Y_{n m}\right\rangle}{\lambda-\beta_{n}} Y_{n m}
$$

and

$$
\phi_{2}=-\sqrt{\frac{4 \pi}{3}} \frac{1}{\lambda-\beta_{1}} Y_{10}
$$

The general solution reads

$$
\psi=-\sqrt{\frac{4 \pi}{3}} \frac{\mu}{\lambda-\beta_{1}} Y_{10}+\sum_{n=1}^{+\infty} \sum_{m=-n}^{n} \frac{\left\langle h \mid Y_{n m}\right\rangle}{\beta_{n}-\lambda} Y_{n m}(\theta, \phi),
$$

or in terms of the potential vorticity

$$
q=\langle h\rangle+\sqrt{\frac{4 \pi}{3}} \frac{\beta_{1}-R^{-2}}{\lambda-\beta_{1}} \mu Y_{10}-\beta \sum_{n=1}^{+\infty} \sum_{m=-n}^{n} \frac{\left\langle h \mid Y_{n m}\right\rangle}{\beta_{n}-\lambda} Y_{n m}(\theta, \phi) .
$$

Defining $\tilde{h}=h+\sqrt{\frac{4 \pi}{3}} \mu Y_{10}$, the stream function takes the compact form

$$
\psi=\sum_{n=1}^{+\infty} \sum_{m=-n}^{n} \frac{\left\langle\tilde{h} \mid Y_{n m}\right\rangle}{\beta_{n}-\lambda} Y_{n m}
$$

The energy is given by the relation

$$
E=-\frac{1}{2}\langle\psi \Delta \psi\rangle+\frac{1}{2} \frac{\left\langle\psi^{2}\right\rangle}{R^{2}}
$$

which gives, after replacing with equations (87) and (88), and simplifying:

$$
E=\frac{\left\langle\phi_{1} \mid \phi_{1}\right\rangle}{8 \pi}\left(R^{-2}-\beta\right)-\frac{\left\langle h \mid \phi_{1}\right\rangle}{8 \pi}+\frac{2 R^{-2}-\beta_{1}}{\left(\lambda-\beta_{1}\right)^{2}} \mu\left(\frac{\mu}{6}+\frac{\left\langle h \mid Y_{10}\right\rangle}{\sqrt{12 \pi}}\right) .
$$

Replacing $\phi_{1}$ with equation (91) - or directly substituting equation (93) in equation 96 - we obtain $E$ as the sum of a series:

$$
E=\frac{1}{8 \pi} \sum_{n=1}^{+\infty} \sum_{m=-n}^{n} \frac{\left|\left\langle\tilde{h} \mid Y_{n m}\right\rangle\right|^{2}}{\left(\beta_{n}-\lambda\right)^{2}}\left(R^{-2}-\beta_{n}\right) .
$$

Similarly, the angular momentum and entropy can be expressed as

$$
\begin{aligned}
& L=-\beta \frac{\left\langle\psi \mid Y_{10}\right\rangle}{\sqrt{12 \pi}}-\frac{\mu}{3}=\frac{1}{3} \frac{\beta_{1}-R^{-2}}{\lambda-\beta_{1}} \mu+\frac{\beta}{\lambda-\beta_{1}} \frac{\left\langle h \mid Y_{10}\right\rangle}{\sqrt{12 \pi}}, \\
& S=-\frac{1}{2}\langle h\rangle^{2}-\frac{\left(\sqrt{4 \pi / 3} \mu\left(\beta_{1}-R^{-2}\right)+\beta\left\langle h \mid Y_{10}\right\rangle\right)^{2}}{8 \pi\left(\beta_{1}-\lambda\right)^{2}}-\frac{\beta^{2}}{8 \pi} \sum_{n, m} \frac{\left|\left\langle h \mid Y_{n m}\right\rangle\right|^{2}}{\left(\beta_{n}-\lambda\right)^{2}},
\end{aligned}
$$

where the sum is on all indices $(n, m)$ except $(0,0)$ and $(1,0)$. 
This is the general solution of the problem. For a given topography, these equations of state determine the Lagrange multipliers $\beta$ and $\mu$ as a function of the energy $E$ and angular momentum $L$. Equation 99 is easily inverted to yield

$$
\mu=\frac{3 \beta}{R^{-2}-\beta_{1}} \frac{\left\langle h \mid Y_{10}\right\rangle}{\sqrt{12 \pi}}-\frac{3\left(\lambda-\beta_{1}\right)}{R^{-2}-\beta_{1}} L .
$$

Similarly, the relation between the Lagrange multiplier $\alpha$ and the circulation $\Gamma$ is easily obtained:

$$
\alpha=-\beta\langle\psi\rangle-\Gamma=-\Gamma=-\langle h\rangle .
$$

From equations (97) and (99), we introduce the control parameter

$$
\mathcal{E}(E, L) \equiv E+3 \frac{2 R^{-2}-\beta_{1}}{\left(R^{-2}-\beta_{1}\right)^{2}} L\left(\frac{\left\langle h \mid Y_{10}\right\rangle}{\sqrt{12 \pi}}-\frac{L}{2}\right),
$$

in terms of which we obtain

$$
\begin{aligned}
& \mathcal{E}=\frac{\left\langle\phi_{1} \mid \phi_{1}\right\rangle}{8 \pi}\left(R^{-2}-\beta\right)-\frac{\left\langle h \mid \phi_{1}\right\rangle}{8 \pi} \\
& +\frac{2 R^{-2}-\beta_{1}}{\left(R^{-2}-\beta_{1}\right)^{2}} \frac{\beta\left(\lambda+R^{-2}-2 \beta_{1}\right)}{\left(\lambda-\beta_{1}\right)^{2}} \frac{\left\langle h \mid Y_{10}\right\rangle^{2}}{8 \pi} .
\end{aligned}
$$

The right hand-side is only a function of $\beta$. Thus, in practice, for a given set of control parameters $(\mathcal{E}, L, \Gamma)$, solving equation (104) for $\beta$ together with equations (101) and (102) yields the value of the Lagrange multipliers $(\beta, \mu, \alpha)$.

If we do not take into account the conservation of angular momentum $(\mu=0)$, equations 97 and 99 reduce to

$$
\begin{aligned}
& E=\frac{\left\langle\phi_{1} \mid \phi_{1}\right\rangle}{8 \pi}\left(R^{-2}-\beta\right)-\frac{\left\langle h \mid \phi_{1}\right\rangle}{8 \pi}, \\
& L=\frac{\beta}{\lambda-\beta_{1}} \frac{\left\langle h \mid Y_{10}\right\rangle}{\sqrt{12 \pi}} .
\end{aligned}
$$

Since $\left\langle\phi_{1} \mid \phi_{1}\right\rangle=\frac{\partial\left\langle h \mid \phi_{1}\right\rangle}{\partial \lambda}$, equation 105 can also be written

$$
E=\frac{1}{8 \pi} \frac{\partial}{\partial \lambda}\left(\left(2 R^{-2}-\lambda\right)\left\langle h \mid \phi_{1}\right\rangle\right) \text {. }
$$

In the case $R=\infty$, this expression reduces to $E=-\frac{1}{2} \frac{d}{d \beta}\left(\beta\left\langle h \phi_{1}\right\rangle\right)$ as found in 63].

\subsection{Degenerate solutions of the mean field equation}

In the case where $\lambda \in \operatorname{Sp} \Delta$, if $\lambda=\beta_{p}$ with $p>1$, then, strictly speaking, we must have $\left\langle h Y_{p m}\right\rangle=0$ for all $-p \leq m \leq p$. In practice, if the topography has a non-vanishing mode of order $(p, m)$, which is the generic case, then as $\lambda \rightarrow \beta_{p}$, the corresponding mode becomes overwhelmingly dominant in the stream function: $\psi \sim \frac{\left\langle h \mid Y_{p m}\right\rangle}{\beta_{p}-\lambda} Y_{p m}$ (if there are several possible values of $m$, then the stream function will be proportional to the appropriate linear combination of these modes). The corresponding divergence for the energy and entropy are like $\left(\lambda-\beta_{p}\right)^{-2}$. If, on the contrary, the topography has no 
contribution proportional to any spherical harmonic of order $p$, then the stream function belongs to the $2 p+1$ dimensional solution space

$$
\psi=\sum_{\substack{n=1 \\ n \neq p}}^{+\infty} \sum_{m=-n}^{n} \frac{\left\langle\tilde{h} \mid Y_{n m}\right\rangle}{\beta_{n}-\beta_{p}} Y_{n m}(\theta, \phi)+\sum_{m=-p}^{p} \phi_{p m} Y_{p m}(\theta, \phi) .
$$

where $\phi_{p m}$ are arbitrary coefficients. The energy and entropy are given by

$$
\begin{aligned}
& E=E\left(\beta_{p}\right)+\frac{1}{2}\left(\frac{1}{R^{2}}-\beta_{p}\right) \sum_{m=-p}^{p} \phi_{p m}^{2}, \\
& S=S\left(\beta_{p}\right)-\frac{\beta_{p}^{2}}{2} \sum_{m=-p}^{p} \phi_{p m}^{2},
\end{aligned}
$$

where it is assumed that in $E\left(\beta_{p}\right)$ and $S\left(\beta_{p}\right)$ the term $n=p$ is discarded in the sum.

As in section 3.2.3 the case $\lambda=\beta_{1}$ is only possible when $\sqrt{\frac{4 \pi}{3}} \mu=\left\langle h \mid Y_{10}\right\rangle$. Then, the equilibrium flow is a superposition between the dipolar flow of section 3.2 .3 and the general continuum solution described in the previous section.

\subsection{Stability of the statistical equilibria}

After some easy algebra, the second order variations of the grand-potential functional $J[q]=S[q]-\beta E[q]-\alpha \Gamma[q]-\mu L[q]$ are proved to be equal to

$$
\delta^{2} J=-\int_{D} \frac{(\delta q)^{2}}{2} d^{2} \mathbf{r}-\frac{\beta}{2} \int_{D}\left((\nabla \delta \psi)^{2}+\frac{(\delta \psi)^{2}}{R^{2}}\right) d^{2} \mathbf{r}
$$

As in section 3.1.3, we see that if $\lambda>\beta_{1}$, the equilibrium flow is a local maximum of the entropy functional.

If there exist a couple $(n, m)$ such that $\left\langle h Y_{n m}\right\rangle=0$, then perturbations proportional to $Y_{n m}$ destabilize the basic flow for $\lambda<\beta_{n}$ while preserving the constraints at first order. But, in general, no coefficient vanishes in the spherical harmonic expansion and it is not sufficient to repeat as it stands the reasoning of section 3.1 .3 with perturbations proportional to eigenvectors of the Laplacian since they would not conserve the energy anymore. However, the orthogonal set of the basic flow remains an hyperplane and one may construct many perturbations not proportional to eigenvectors of the Laplacian. To keep the computation of the second order variations of the free energy with respect to the perturbation simple, we shall look for a destabilizing perturbation in a twodimensional subspace of this hyperplane. Let us consider the perturbations spanned by two eigenvectors of the Laplacian: $\delta \psi=\epsilon_{1} Y_{n_{1} m_{1}}+\epsilon_{2} Y_{n_{2} m_{2}}$. Clearly the corresponding variation of the potential vorticity is $\delta q=\epsilon_{1}\left(R^{-2}-\beta_{n_{1}}\right) Y_{n_{1} m_{1}}+\epsilon_{2}\left(R^{-2}-\beta_{n_{2}}\right) Y_{n_{2} m_{2}}$. For any non-zero $n_{1}, n_{2}$, this perturbation conserves the circulation. The variation of the energy is found to be

$$
\delta E=\epsilon_{1}\left(R^{-2}-\beta_{n_{1}}\right) \frac{\left\langle h Y_{n_{1} m_{1}}\right\rangle}{\beta_{n_{1}}-\lambda}+\epsilon_{2}\left(R^{-2}-\beta_{n_{2}}\right) \frac{\left\langle h Y_{n_{2} m_{2}}\right\rangle}{\beta_{n_{2}}-\lambda} .
$$


To ensure that the perturbation conserves the energy, we choose $\epsilon_{2}$ such that $\delta \psi$ is orthogonal to the basic flow:

$$
\epsilon_{2}=-\epsilon_{1} \frac{\left(R^{-2}-\beta_{n_{1}}\right)\left(\beta_{n_{2}}-\lambda\right)\left\langle h Y_{n_{1} m_{1}}\right\rangle}{\left(R^{-2}-\beta_{n_{2}}\right)\left(\beta_{n_{1}}-\lambda\right)\left\langle h Y_{n_{2} m_{2}}\right\rangle} .
$$

It remains to see if the main flow is stable or not against this perturbation. To that purpose we need to compute $\delta^{2} J$. We find that

$$
\delta^{2} J=-\left(\epsilon_{1}^{2}\left(R^{-2}-\beta_{n_{1}}\right)\left(\lambda-\beta_{n_{1}}\right)+\epsilon_{2}^{2}\left(R^{-2}-\beta_{n_{2}}\right)\left(\lambda-\beta_{n_{2}}\right)\right) / 2,
$$

and with the particular choice 113 for $\epsilon_{2}$, we obtain the criterion:

$$
\delta^{2} J \geq 0 \Longleftrightarrow f(\lambda) \leq 0
$$

with

$$
\begin{aligned}
& f(\lambda)=\left|\left\langle h Y_{n_{2} m_{2}}\right\rangle\right|^{2}\left(R^{-2}-\beta_{n_{2}}\right)^{2}\left(\lambda-\beta_{n_{1}}\right)^{3} \\
& +\left|\left\langle h Y_{n_{1} m_{1}}\right\rangle\right|^{2}\left(R^{-2}-\beta_{n_{1}}\right)^{2}\left(\lambda-\beta_{n_{2}}\right)^{3} .
\end{aligned}
$$

From the computation of its derivative, we can see that $f$ is a monotonically increasing function of $\lambda$. Let us define

$$
\kappa^{2}=\frac{\left(R^{-2}-\beta_{n_{2}}\right)}{\left(R^{-2}-\beta_{n_{1}}\right)} \frac{\left|\left\langle h Y_{n_{2} m_{2}}\right\rangle\right|^{2}}{\left|\left\langle h Y_{n_{1} m_{1}}\right\rangle\right|^{2}},
$$

Then, $f$ vanishes for $\lambda^{*}=\left(\beta_{n_{2}}+\kappa^{2 / 3} \beta_{n_{1}}\right) /\left(1+\kappa^{2 / 3}\right)$. The instability condition thus reads

$$
\delta^{2} J \geq 0 \Longleftrightarrow \lambda \leq \lambda^{*}
$$

Clearly, $\lambda^{*}$ lies in between $\beta_{n_{1}}$ and $\beta_{n_{2}}$, and for $n_{1}=n_{2}=n, \lambda^{*}=\beta_{n}$. In particular the choice $n_{1}=n_{2}=1$ (with $m_{1} \neq m_{2}$ ) proves that all the solutions with $\lambda<\beta_{1}$ are saddle points.

\subsection{The effect of the bottom topography}

We now turn to the description of the influence of an arbitrary topography and a finite Rossby deformation radius on the structure of the statistical equilibria. As the effect of angular momentum was already studied thoroughly in section 3.2 , we shall assume in the sequel that $\mu=0$.

The interpretation of relation (93) linking the topography (including the Coriolis effect) and the equilibrium streamfunction is relatively simple. The general structure of the topography is reproduced in the equilibrium flow, with a statistical temperaturedependent weighing of each mode. In particular, the modes of the topography with eigenvalues close to the statistical temperature will prevail in the final flow. In general, for a given statistical temperature, the small-scale details of the topography (i.e. highorder modes) will not affect the general structure of the equilibrium flow: their amplitude in the equilibrium streamfunction decreases relatively quickly, as $1 / n^{2}$ where $n$ is the order of the (degenerate) mode. 

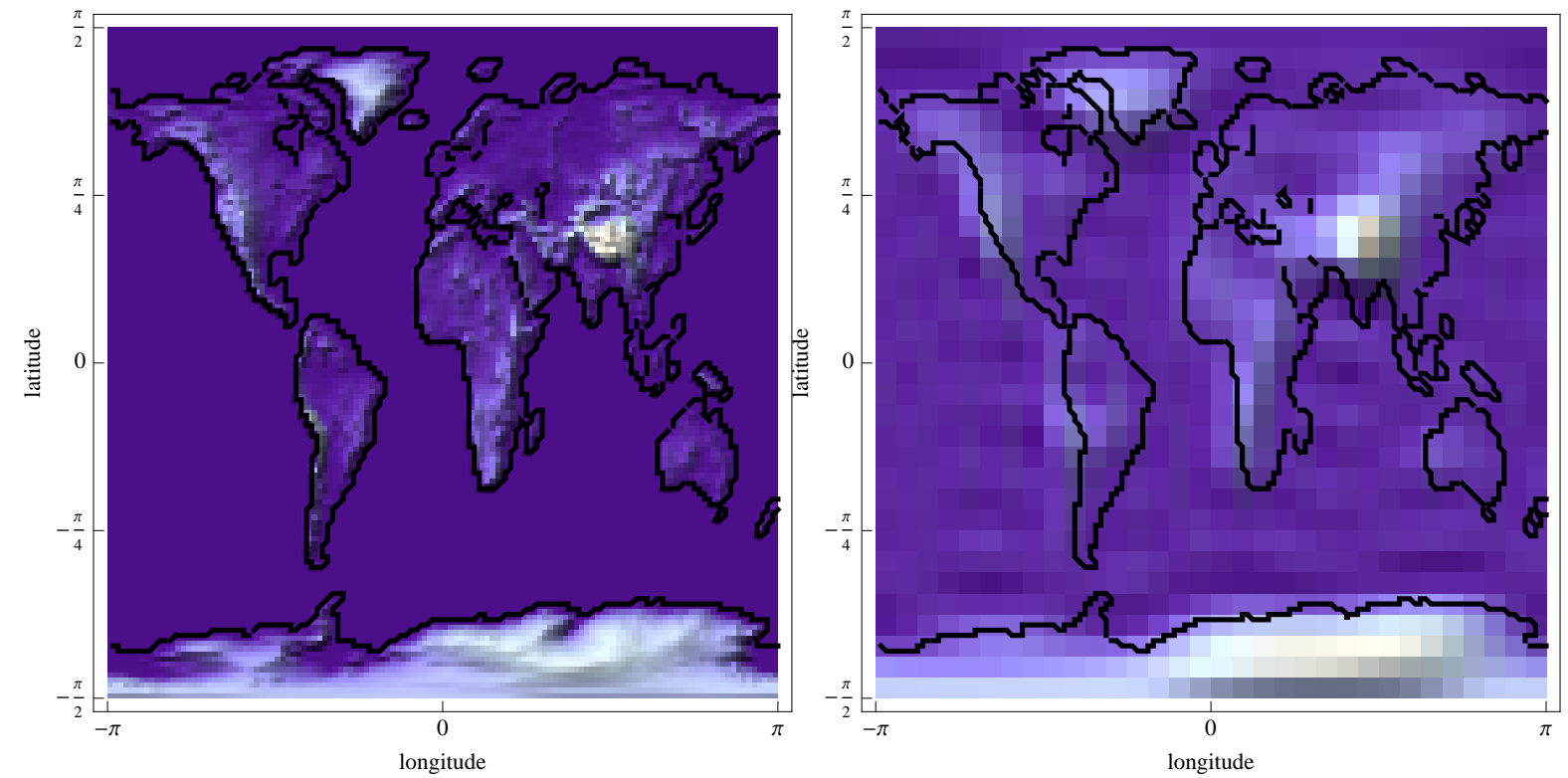

Figure 11. The Earth's bottom topography (left) and its T16 spectral truncation (right).

For a more detailed discussion, we consider a specific example with the Earth's topography in a T16 spectral truncation (see figure 11). Figure 12 shows the caloric curve $\beta(E)$ for this topography in the limit of an infinite Rossby deformation radius $(R=\infty)$. This curve essentially consists of spikes at eigenvalues of the Laplacian superimposed on a background curve similar to the caloric curve obtained in the absence of topography (figure 1). For every value of the energy, there exist an infinity of possible statistical temperatures, but only one corresponds to an entropy maximum (the one corresponding to $\beta>\beta_{1}$ ). All the other solutions are saddle points of the entropy functional, but one can imagine that they are only marginally unstable, so that the system may remain stuck in these states for a long time. The robustness of these saddle points has been illustrated by Naso et al. [65] who stressed their importance. These semi-persistent states could be relevant for climate modeling. Indeed, the atmosphere is never in a permanent equilibrium state. If we find large-scale structures that are, strictly speaking, unstable but whose lifetime is, however, of the order of a few days, such structures are fully relevant for climate modeling. They could account for situations of atmospheric blocking. For this reason, we shall comment on several critical points of the entropy functional (indicated with red dots on the caloric curve of figure 12 in cases of high (points $H 1, H 2, H 3, H 4)$ and low energy $(L 1, L 2, L 3, L 4)$ regardless of the nature of these critical points.

At high energy, possible steady states for the flow are basically just the degenerate modes. From the stability analysis of previous section, we know that among all these modes, the only stable solution is the one with $\beta \approx \beta_{1}$. Nevertheless, saddle points corresponding to $\beta \approx \beta_{n}$ can be long-lived as the system may not generate spontaneously the perturbations that destabilize them. However, the bigger $n$ is, the more such 


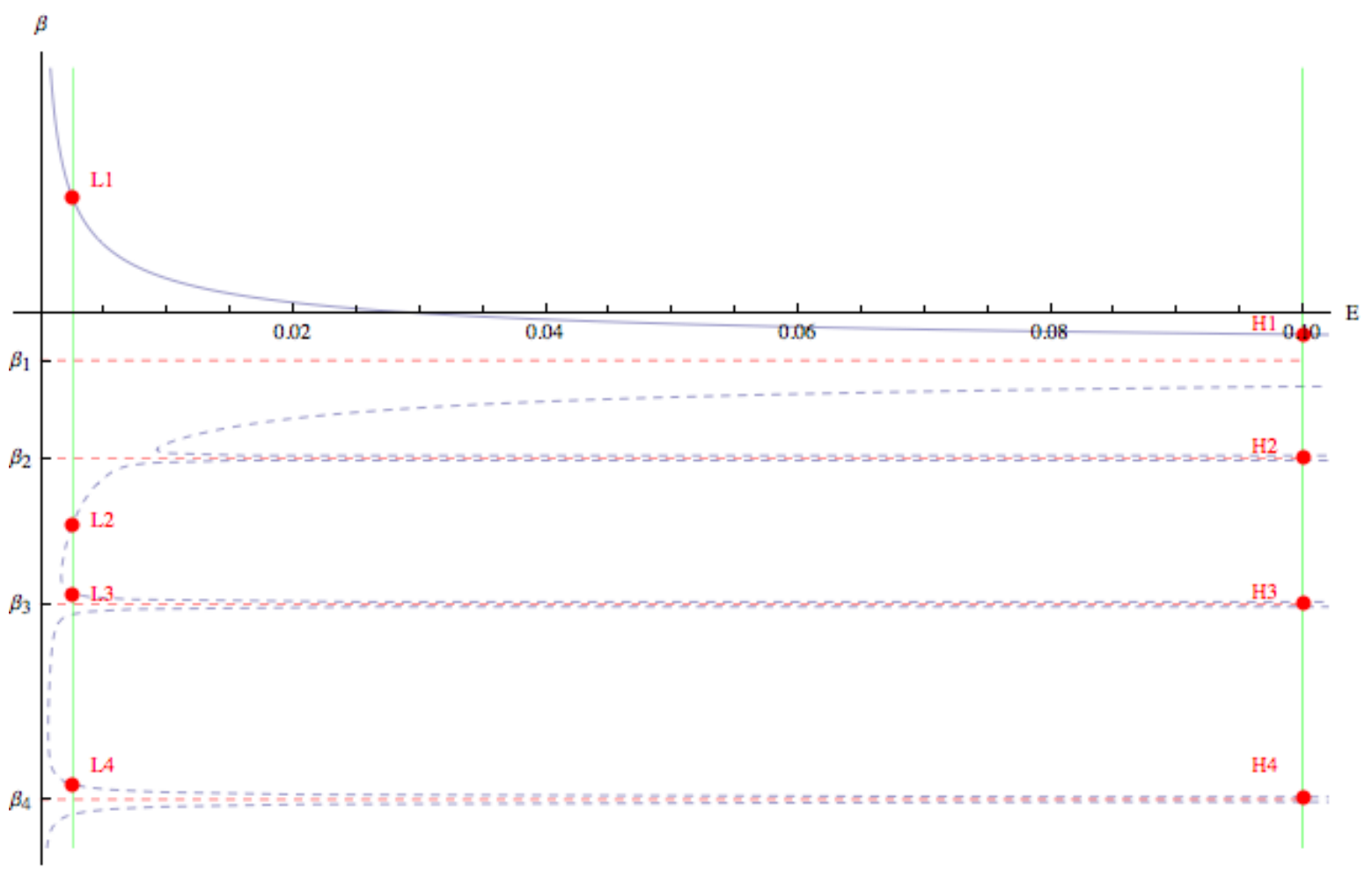

Figure 12. The caloric curve $\beta(E)$ for the Earth's topography in T16 spectral truncation, with $R=\infty$. The solid blue line corresponds to entropy maxima while the solutions represented with dashed blue lines are saddle points. The position of the eigenvalues of the Laplacian is marked with red dashed lines. We show exemples of high energy flows $H 1, H 2, H 3, H 4$ in figure 13 and exemples of low-energy flows $L 1, L 2, L 3, L 4$ in figure 14 .

perturbations exist. Thus, one can expect that eigenmodes with "large" $n$ will be less stable than eigenmodes with "low" $n$. We have plotted on figure 13 examples of equilibrium streamfunctions for high-energy flows with the Earth's topography (the first four modes are represented). For $\beta \approx \beta_{1}$, we obtain the well-known solid-body rotation. The saddle solution for $\beta \approx \beta_{2}$ is a quadrupole, while the states with $\beta \approx \beta_{3}$ and $\beta \approx \beta_{4}$ (also saddles) feature vortices belts in the middle latitudes.

At low energy, the inverse temperature $\beta$ is not constrained to be in the vicinity of a Laplacian eigenvalue anymore. As a consequence, the structure of the flow is less constrained and consists of a mixing of many different modes. As examples, the equilibrium stream functions for $\beta=4.7,-8.7,-11.6$ and -19.4 are given in figure 14 with the Earth's topography. Note that, as explained before, the background solid body rotation has a different sign depending on the sign of $\beta-\beta_{1}$. Similarly to the high energy case, the solutions with $\beta<\beta_{1}$ are formally unstable, but it is possible that the system remains stuck in these states for a long time.

For the above discussion, we have chosen to use the topography of the Earth to fix ideas, but in fact the qualitative features of the flow examined here do not depend much on this particular choice. We have also considered a dummy topography with 

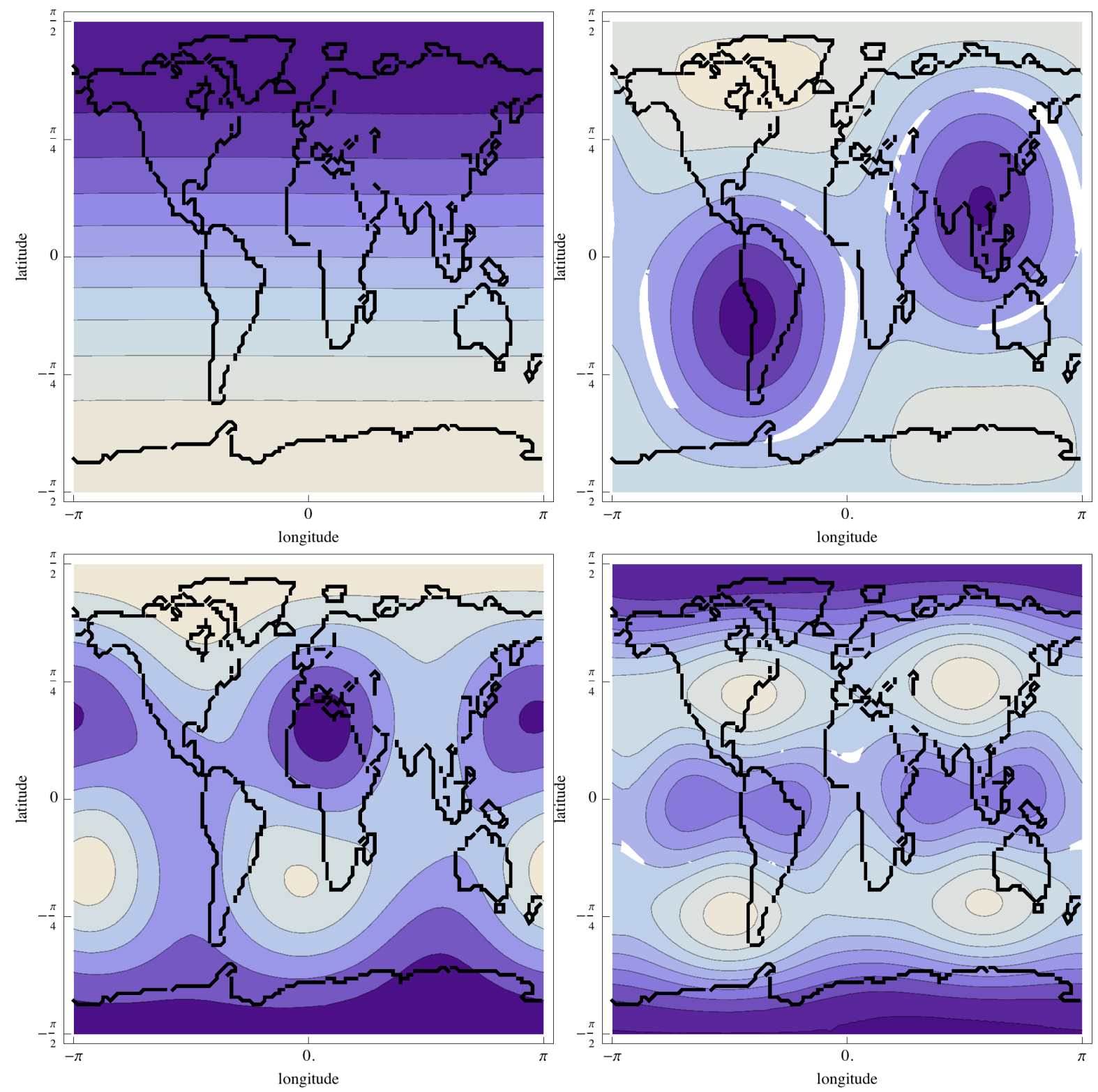

Figure 13. High energy $(E=1)$ flows: Equilibrium stream function for $\beta \approx \beta_{1}$, $\beta \approx \beta_{2}, \beta \approx \beta_{3}$ and $\beta \approx \beta_{4}$ with the Earth's topography in T16 spectral truncation, with $R=\infty$. From top to bottom and left to right, these flows are the $H 1, H 2, H 3$ and $H 4$ flows from figure 12 .

the same truncation (T16) but with uniform spectra, retaining the same total power as the topography of the Earth. The resulting caloric curve is plotted on figure 15 and compared to the caloric curve obtained with the topography of the Earth. In the high energy range, the difference is not very important. Figure 15 also shows $\beta$ as a function of $1 / E$ to make apparent the differences that occur in the low energy range. The main difference between the two curves is found in the vicinity of $\beta_{1}$, as expected from the strong Coriolis domination for the topography of the Earth. As shown in figure 15 (right), the bottom topography plays an important role at low energies, 

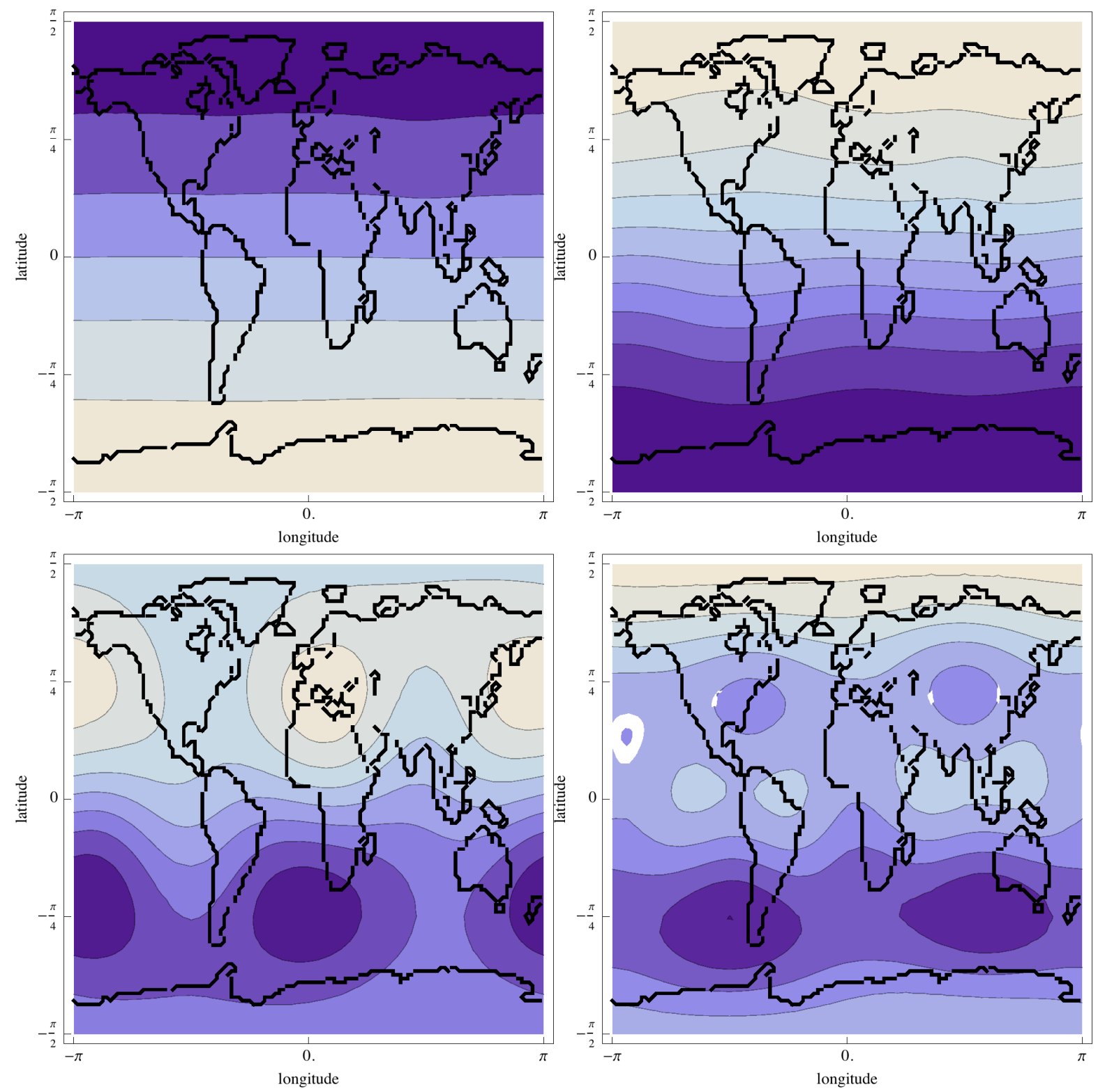

Figure 14. Low energy flows $(E \approx 0.0026)$ : Equilibrium stream function for $\beta \approx 4.7$, $\beta \approx-8.7, \beta \approx-11.6$ and $\beta \approx-19.4$ with the Earth's topography in T16 spectral truncation, with $R=\infty$. From top to bottom and left to right, these flows are the $L 1, L 2, L 3$ and $L 4$ flows from figure 12 .

similarly to Fofonoff flows [63]. Actually, the structure of the stable equilibrium does not depend much on the topography, even at low energy. On the contrary, the unstable structures obtained as saddle points of the entropy, even if they always exist, may not be reached by a flow with low energy if the contribution of the topography is not strong enough. On figure 15 (right), it is clear that the branches between two eigenvalues of the Laplacian extend more or less towards low energies depending on the topography. As an illustration, for the energy of our low-energy flows L1,L1,L3,L4, the quadrupole state is not possible. 

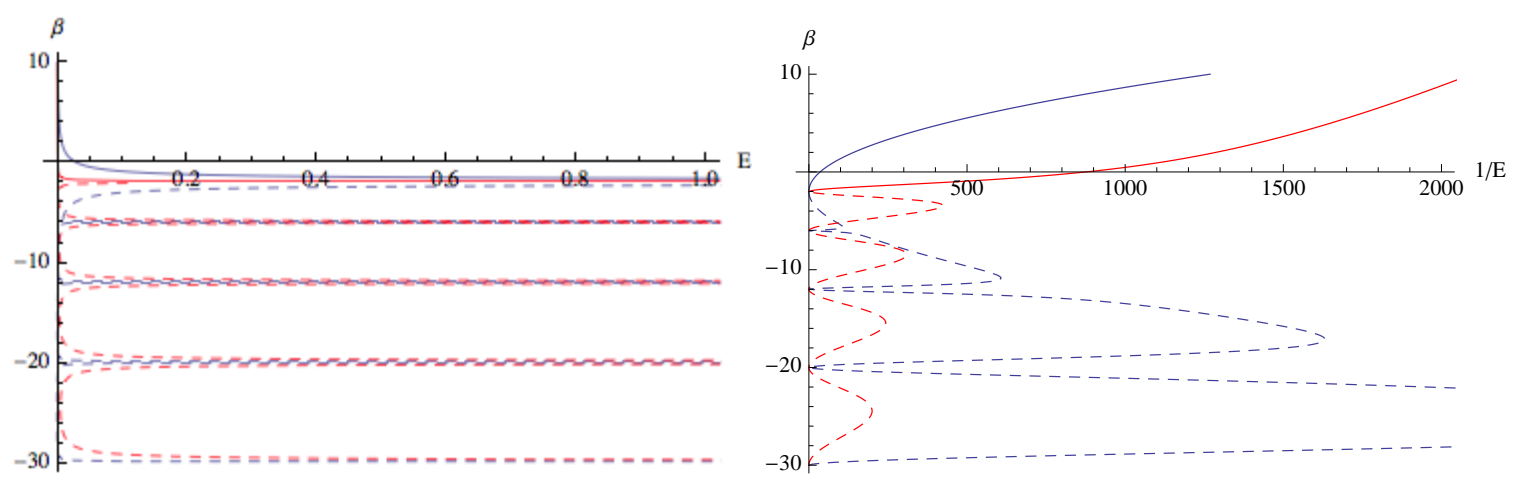

Figure 15. Caloric curve $\beta(E)$ (left) and $\beta(1 / E)$ (right) with $R=\infty$, for the Earth's topography in a T16 truncation (blue) and for a dummy topography with uniform spectrum and the same total power as the Earth's topography (red). The curve $\beta(E)$ (left) clearly shows that the influence of the topography is small at high energies as the statistical temperature is very close to Laplacian eigenvalues regardless of the topography. On the contrary, at low energy, the details of the topography are important, especially to determine which saddle points can be attained by the system (right).

In fact, the low-energy limit can be understood with a simple approximation. When $\beta \rightarrow \infty$ (or equivalently, when $E \rightarrow 0$ ), it is possible to neglect the Laplacian (except in boundary layers but there is none in our spherical geometry) in the mean-field equation $\Delta \psi-\lambda \psi=\alpha+h+\mu \cos \theta$. We obtain $\lambda \psi=-\alpha-h-\mu \cos \theta$, which means that the flow is a superposition of a solid-body rotation term $-(\mu / \lambda) \cos \theta$ due to the conservation of the angular momentum, and a term $-h / \lambda$ directly fixed by the topography. Again, this is similar to the case of Fofonoff flows studied in [63].

\subsection{The role of the Rossby deformation radius}

In the previous section, we have analyzed the effect of arbitrary topographies on the equilibrium states, but only in the limit of infinite Rossby deformation radius. In fact, the mean field equation for a finite Rossby deformation radius is the same as that for infinite $R$ if we make the change of variable for the statistical temperature $\lambda=\beta+1 / R^{2}$. As a consequence, the set of equilibrium states in itself is unchanged. Indeed, as equation (93) shows, the set of stream function spanned as we vary $\lambda$ is the same as that obtained by varying $\beta$ in the limit $R=+\infty$. On the contrary, the expressions for the energy (equation (98) ) and entropy (equation (100) ) depend directly on $R$ and not only on $\lambda$. Figure 16 compares the caloric curve obtained for $R=\infty$ and for $R=1$ (with $\langle\psi\rangle=0$ or, equivalently, $\Gamma=\langle h\rangle$ ). The main effect of the finite Rossby deformation radius is to shift the curve towards negative statistical temperature values, as expected from the identity $\lambda=\beta+1 / R^{2}$. The qualitative picture discussed above is not modified by the Rossby deformation radius if we replace everywhere $\beta$ by $\lambda$. Note that for a fixed statistical temperature $\beta$, the energy is generally higher for a finite Rossby deformation radius. 


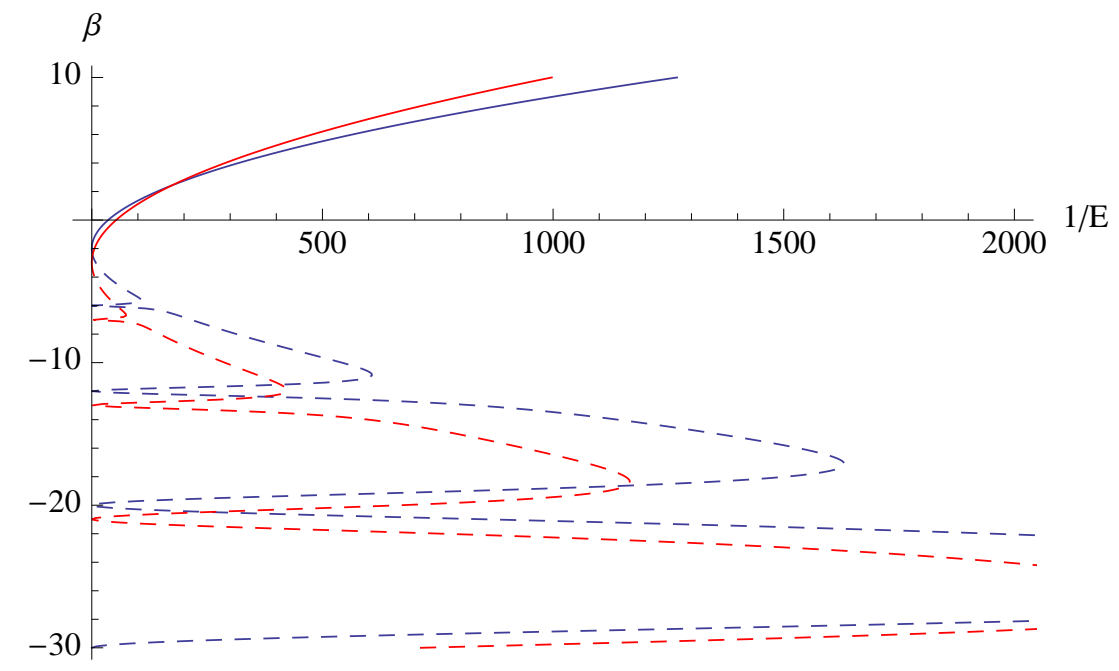

Figure 16. Caloric curve $\beta(1 / E)$ for the Earth's topography in a T16 spectral truncation, with infinite Rossby deformation radius (blue) and with $R=1$ (red). The effect of the finite Rossby deformation radius is mainly seen for low values of the energy.

\section{Discussion}

Let us summarize the results obtained in the previous sections. The statistical equilibrium states (in the sense as those corresponding to a linear $q-\psi$ relationship) of the quasi-geostrophic equations with conservation of energy and circulation on a rotating sphere are as follows:

- For a smooth (without bottom topography) sphere without taking into account the conservation of the vertical component of the angular momentum: the only stable state is a counter-rotating solid-body rotation. There is always an unstable equilibrium state which is a co-rotating solid-body rotation. Depending on the energy, one may also have unstable degenerate eigenmodes (since the coefficients are undetermined, these can be any combination of the corresponding spherical harmonics), which are saddle points of the entropy.

- For a smooth sphere taking into account conservation of the vertical component of the angular momentum, there are two possibilities: if the energy and angular momentum values satisfy the relation $E=E^{*}(L)$, we have a solid-body rotation with angular velocity $\Omega_{*}=3 L / 2$. In particular, both counter-rotating and corotating rotations are possible as stable equilibria. Otherwise, the statistical equilibrium is a dipole: in this case, we observe a spontaneous symmetry breaking associated with a second order phase transition. Again there are possibilities of unstable saddle points states as in the previous case.

- For a sphere with a bottom topography, the equilibrium states are unchanged, but there are extra saddle points states featuring higher-order spherical harmonics $(n \geq 2)$ that are more constrained (due to the topography) than when they appear 
as degenerate solutions of the Helmholtz mean-field equation. At high energies, the topography has a negligible effect, while it becomes important at low energies.

One of the original motivations of the theories of statistical mechanics for twodimensional turbulent flows [75, 80] was to account for the observed tendency for the flow to organize into coherent structures, such as monopoles, dipoles, tripoles, quadrupoles, etc $([1,94$, 95, 96, 97, 98, 99, 100, 2, 101]). The numerical simulations suggesting these large-scale structures, as well as the theoretical attempts to obtain them as statistical equilibria, focus on planar flow cases with either fixed or periodic boundary conditions, and flows in a $\beta$-channel [102]. In the present study, we find that the combined effect of rotation and spherical geometry partially annihilates these coherent structures: in the absence of a bottom topography, the equilibrium flow is purely zonal. This prediction from the theory is confirmed by early results obtained in numerical simulations of 2D turbulent flows on a sphere [103, 104, 105, 106]. However, if we impose an additional constraint of angular momentum conservation, we recover a dipolar equilibrium state (for some values of the external parameters). Interestingly, in this case the statistical mechanics presents a spontaneous symmetry-breaking feature: we obtain a set of equilibrium states with a phase factor as a free parameter. For each particular equilibrium state, the axial symmetry is broken but the action of rotations around the vertical axis leaves the set of equilibrium states globally invariant. This spontaneous symmetry breaking appears due to the presence of a second order phase transition, which occurs in both the microcanonical and the grand-canonical ensembles. It is also noteworthy that the thermodynamical properties of the system are quite unusual [64]. The microcanonical and grand-canonical ensembles are equivalent, but only marginally so: the entropy functional is a plane and, as such, it is concave but it is also convex. The statistical temperature does not depend on the energy in the dipole phase, and can be anything greater than $\beta_{1}$ in the solid-body rotation phase. Besides, the specific heats $\partial^{2} \mathcal{S} / \partial E^{2}$ and $\partial^{2} \mathcal{S} / \partial L^{2}$ both vanish.

In direct simulations on a non-rotating sphere, conservation of all the components of angular momentum leads to the formation of coherent structures, while in the presence of rotation, when only one component of the angular momentum is conserved, zonal structures emerge. In fact, carrying out the statistical mechanics procedure developed in this study with all the components of the angular momentum conserved (and thus vanishing rotation) indeed yields coherent structures similar to those observed in numerical simulations. The two extra conserved quantities add terms proportional to $\sin \theta \cos \phi$ and $\sin \theta \sin \phi$, which lead to a flow similar to the dipole obtained in section 3.2 .3 , except that the phase is now fixed by the angular momentum constraint. Besides, this flow perdures for external parameters varying in a wide range, whereas it only occurs for $\beta=\beta_{1}$ and $\mu=-2 \Omega$ in section 3.2 .3 .

On the rotating sphere, as summarized above, the stable equilibrium flow does not present complex vortices, except in one case if we take into account conservation of angular momentum. But as shown on figure 13 in the presence of a bottom topography, many coherent structures can be realized as unstable saddle points states with a 
statistical temperature close to a Laplacian eigenvalue. Those corresponding to loworder eigenvalues (like the quadrupole) are likely to be only weakly unstable, since only low-order perturbations can destabilize them. They could account for atmospheric blocking where a vortex persists for a few days and is finally destabilized and disappears.

Finally, also note that even in the absence of a bottom topography and without conservation of angular momentum, coherent vortices can be obtained as degenerate modes $(\beta \in \operatorname{Sp} \Delta)$. The resulting stream function then resembles the examples in figure 13, except that the coefficients are not determined in any way. Thus, virtually any combination of the eigenvectors for this eigenvalue is an acceptable equilibrium stream function. However, as pointed out before, these states are not stable, even though they can be long-lived, especially low-order modes.

It would be of great interest to compute the statistical equilibrium obtained from the theory for realistic values of the constraints (kinetic energy, angular momentum) and to compare it to observations and predictions from dynamical models. In the case of the Earth, this was done by Verkley and Lynch [56] in the framework of the Kraichan energy-enstrophy theory for a spectrally truncated model, with partial agreement. We shall report elsewhere the results obtained by doing so with the theory developed here. Note that [56] considers some simple representation of forcing and dissipation. Although the results obtained are very encouraging, it is difficult to justify rigorously the inclusion of forcing and dissipation, as the Liouville theorem does not automatically hold in this case. However, in real flows, forcing and dissipation do not equilibrate locally, and they play an important role in the theory of geophysical fluids. Nevertheless, there are some known cases where quasi-stationnary states reached by a forced-dissipated system are well approximated by some equilibrium states of a system with no forcing and no dissipation. An important example is the von Karman flow [47, 31. This may indicate that, at least in some cases, the non-equilibrium attractors may remain in the vicinity of some equilibrium states. However, the general question of the relevance of equilibrium approaches to non-equilibrium problems is far from being understood.

\section{Conclusion}

In this paper, we have applied the Miller-Robert-Sommeria statistical theory for perfect inviscid fluids to the general circulation on a rotating sphere. The large-scale circulation is modelled by a one-layer (barotropic) quasi-geostrophic flow and the potential vorticity plays the role of the vorticity in the MRS theory. If we only consider the conservation of the fine-grained enstrophy among the infinite class of Casimirs, the maximization of the MRS entropy at fixed energy, circulation, angular momentum and fine-grained enstrophy is equivalent, for what concerns the mean field, to the minimization of the coarse-grained enstrophy at fixed energy, circulation and angular momentum [65]. This leads to a linear $q-\psi$ relationship. Furthermore, the fluctuations around the mean field state are gaussian. We have shown that the mean field equation can be solved analytically in a very simple way due to the geometry of the domain and the fact that 
the Coriolis parameter is an eigenvector of the Laplacian on the sphere. Ignoring the conservation of angular momentum, we have found that the stable statistical equilibrium flow is a counter-rotating solid-body rotation. Taking the conservation of angular momentum into account, we have obtained two qualitatively different equilibrium flows: solid-body rotation (reminiscent of the previous case) or dipole. In the latter case, the axial symmetry is spontaneously broken and the system displays a second order phase transition. Finally, we have shown that the equilibrium quasi-geostrophic flow on a sphere with arbitrary bottom topography has the same background structure with topography-induced modes superimposed. In particular, unstable saddle points modes with multipole vortex structures appear, even though they were already possible as degenerate modes in the absence of a bottom topography. Albeit obtained with a relatively simple model, these results suggest that statistical mechanics constitutes a nice and efficient framework for theoretical studies of large-scale planetary circulation. They also provide a strong incentive to generalize the statistical mechanical methods to more realistic models of the atmosphere and oceans.

\section{Appendix A. Solid-body rotations}

There is a specific type of flow which is of central importance in this study: solid-body rotations. These correspond to the case when all the fluid revolves around the axis of rotation of the sphere, exactly as if it were a solid. Under these conditions, the stream function reads

$$
\psi=\Omega_{*} \cos \theta,
$$

where $\Omega_{*}$ is the angular velocity of the solid-body rotation. Clearly for this specific flow, all the dynamical invariants are not independent, as all the dynamical quantities depend only on $\Omega_{*}$. In fact, straightforward computations lead to

$$
\begin{aligned}
E & =\frac{\Omega_{*}^{2}}{3}, \\
L & =\frac{2}{3} \Omega_{*}, \\
\Gamma_{2} & =\frac{1}{3}\left(\Omega+\Omega_{*}\right)^{2} .
\end{aligned}
$$

We thus introduce the function $E^{*}(L)=3 L^{2} / 4$ which gives the energy of a solid-body rotation with angular momentum $L$. In Appendix B, we show that the energy of any flow on the sphere is always greater than the energy of a solid-body rotation with the same angular momentum. Alternatively, we can define the functions $L_{+}^{*}(E)=\sqrt{4 E / 3}$ and $L_{-}^{*}(E)=-\sqrt{4 E / 3}$. The angular momentum of a solid-body rotation in the positive direction with energy $E$ is $L_{+}^{*}(E)$, while $L_{-}^{*}(E)$ is the angular momentum of a solidbody rotation in the negative direction with energy $E$. Clearly, specifying the energy of the solid-body rotation is not enough, one needs to know in addition the direction of rotation, hence the two functions $L_{+}^{*}$ and $L_{-}^{*}$. 
Note that the relation between the energy, the angular momentum, and the enstrophy which arises as the thermodynamic equilibrium entropy in the text is already fixed by the dynamical constraints in the case of a solid-body rotation: $-\Gamma_{2} / 2=$ $-2 \Omega^{2} / 3-2 E-2 \Omega L$.

\section{Appendix B. Minimum energy for a flow with given angular momentum}

For quasi-geostrophic flows on a rotating sphere, the kinetic energy reads $E=\left\langle u^{2}+v^{2}\right\rangle / 2$ where $u$ and $v$ are the components of the velocity. The vertical component of the angular momentum is $L=\langle u \sin \theta\rangle=\langle(q-f) \cos \theta\rangle$. The Cauchy-Schwarz inequality immediately yields

$$
\left(\int_{S^{2}} u \sin \theta d S\right)^{2} \leq \frac{8 \pi}{3}\left(\int_{S^{2}} u^{2} d S\right)
$$

and consequently,

$$
E \geq \frac{3}{4} L^{2}
$$

Therefore, we always have $E \geq E^{*}(L)$. The lower bound for the energy is reached for a solid-body rotation (see Appendix A). We can also say that, for a given energy $E$, the angular momentum $L$ must satisfy $L_{-}^{*}(E) \leq L \leq L_{+}^{*}(E)$.

Another derivation of this inequality sheds more light on its physical interpretation. For a given value of the angular momentum $L$, it is always possible to find a reference frame in which $L^{\prime}=0$. Indeed, in a reference frame $\mathcal{R}^{\prime}$ rotating with angular velocity $\Omega^{\prime}$ relative to the Earth's rotation, straightforward computations give

$$
\begin{aligned}
& L^{\prime}\left(\Omega^{\prime}\right)=L-\frac{2}{3} \Omega^{\prime}, \\
& E^{\prime}\left(\Omega^{\prime}\right)=E-L \Omega^{\prime}+\frac{1}{3} \Omega^{\prime 2},
\end{aligned}
$$

where $E^{\prime}$ is the energy in $\mathcal{R}^{\prime}$. Clearly, the value of $\Omega^{\prime}$ such that $L^{\prime}=0$ is $\Omega^{\prime}=\frac{3}{2} L$, and it is also the value for which $E^{\prime}$ is a minimum. Thus

$$
E^{\prime}\left(\frac{3}{2 L}\right)=E-\frac{3}{4} L^{2} \geq 0
$$

so that we again obtain $E \geq E^{*}(L)$.

Finally, the shortest way to this inequality is perhaps to decompose the fields on spherical harmonics so that

$$
\omega=\sum_{n=0}^{+\infty} \sum_{m=-n}^{n} \omega_{n m} Y_{n m}, \quad \psi=\sum_{n=0}^{+\infty} \sum_{m=-n}^{n} \frac{\omega_{n m}}{\beta_{n}} Y_{n m}
$$

and

$$
E=-\frac{1}{8 \pi} \sum_{n=0}^{+\infty} \sum_{m=-n}^{n} \frac{\left|\omega_{n m}\right|^{2}}{\beta_{n}}
$$


Clearly, $L=\left\langle\omega \mid Y_{10}\right\rangle / \sqrt{12 \pi}=\omega_{10} / \sqrt{12 \pi}$ and $E \geq\left|\omega_{10}\right|^{2} /\left(-8 \pi \beta_{1}\right)$ so that, again, $E \geq E^{*}(L)$. This proof makes it evident that the inequality only means that the energy is always at least the energy contained in the solid-body rotation mode, which is directly fixed by the angular momentum.

For fixed enstrophy, the classical Fjortoft argument [107] also gives an upper bound on the energy:

$$
\Gamma_{2}=4 \Omega L+\frac{4}{3} \Omega^{2}+\frac{1}{4 \pi} \sum_{n, m}\left|\omega_{n m}\right|^{2},
$$

so that $E \leq \sum_{n, m}\left|\omega_{n m}\right|^{2} /\left(-8 \pi \beta_{1}\right)=\left(\Gamma_{2}-4 \Omega L-4 \Omega^{2} / 3\right) / 4$. Finally,

$$
\frac{3}{4} L^{2} \leq E \leq \frac{\Gamma_{2}}{4}-\Omega L-\frac{\Omega^{2}}{3},
$$

where all the inequalities become equalities in the case of a solid-body rotation. 


\section{References}

[1] McWilliams J. C, 1984, J. Fluid Mech., 14621.

[2] Santangelo P, Benzi R and Legras B, 1989, Phys. Fluids A, 11027.

[3] Couder Y and Basdevant C, 1986, J. Fluid Mech., 173225.

[4] Nguyen-Duc J and Sommeria J, 1988, J. Fluid Mech., 192175.

[5] van Heijst G. J. F and Kloosterziel R. C, 1989, Nature, 338569.

[6] van Heijst G. J. F and Flor J. B, 1989, Nature, 340212.

[7] Onsager L, 1949, Il Nuovo Cimento, 6279.

[8] Montgomery D and Joyce G, 1974, Phys. Fluids, 171139.

[9] Kida S, 1975, J. Phys. Soc. Jpn, 391395.

[10] Pointin Y and Lundgren T, 1976, Phys. Fluids, 191459.

[11] Lundgren T and Pointin Y, 1977, J. Stat. Phys., 17323.

[12] Fröhlich J and Ruelle D, 1982, Commun. Math. Phys., 871.

[13] Benfatto G, Picco P and Pulvirenti M, 1987, J. Stat. Phys., 46729.

[14] Joyce G and Montgomery D, 1973, J. Plasma Phys., 10107.

[15] Smith R and O'Neil T, 1990, Phys. Fluids B, 22961.

[16] Chavanis P.-H. Statistical mechanics of two-dimensional vortices and stellar systems. In Dauxois T, Ruffo S, Arimondo E and Wilkens M, editors, Dynamics and thermodynamics of systems with long-range interactions, volume 602 of Lecture Notes in Physics, pages 208-289. Springer, 2002.

[17] Eyink G and Sreenivasan K, 2006, Rev. Mod. Phys., 7887.

[18] Lee T. D, 1952, Quarterly of Applied Mathematics, 1069.

[19] Kraichnan R, 1967, Phys. Fluids, 101417.

[20] Kraichnan R, 1975, J. Fluid Mech., 67155.

[21] Deem G and Zabusky N, 1971, Phys. Rev. Lett., 27396.

[22] Basdevant C and Sadourny R, 1975, J. Fluid Mech., 69673.

[23] Chorin A, 1994, Vorticity and Turbulence. Springer: New-York.

[24] Miller J, 1990, Phys. Rev. Lett., 652137.

[25] Miller J, Weichman P and Cross M, 1992, Phys. Rev. A, 452328.

[26] Robert R and Sommeria J, 1991, J. Fluid Mech., 229291.

[27] Robert R, 1991, J. Stat. Phys., 65531.

[28] Michel J and Robert R, 1994, Commun. Math. Phys., 159195.

[29] Robert R, 2000, Commun. Math. Phys., 212245.

[30] Lynden-Bell D, 1967, Mon. Not. R. Astron. Soc., 136101.

[31] Monchaux R, Ravelet F, Dubrulle B, Chiffaudel A and Daviaud F, 2006, Phys. Rev. Lett., 96 124502.

[32] Thess A, Sommeria J and Jüttner B, 1994, Phys. Fluids, 62417.

[33] Jüttner B, Thess A and Sommeria J, 1995, Phys. Fluids, 72108.

[34] Tabeling P, 2002, Phys. Rep., 3621.

[35] Dauxois T, Ruffo S, Arimondo E and Wilkens M, editors, 2002, Dynamics and Thermodynamics of Systems with Long Range Interactions, volume 602 of Lecture Notes in Physics. Springer, New-York.

[36] Ellis R, Haven K and Turkington B, 2000, J. Stat. Phys., 101999.

[37] Ellis R, Haven K and Turkington B, 2002, Nonlinearity, 15239.

[38] Bouchet F and Barre J, 2005, J. Stat. Phys., 1181073.

[39] Venaille A and Bouchet F, 2009, Phys. Rev. Lett., 102104501.

[40] Venaille A and Bouchet F, 2011, J. Stat. Phys., 143346.

[41] Chavanis P.-H., 2006, Int. J. Mod. Phys. B, 203113

[42] Ravelet F, Marié L, Chiffaudel A and Daviaud F, 2004, Phys. Rev. Lett., 93164501.

[43] Naso A, Thalabard S, Collette G, Chavanis P.-H and Dubrulle B, 2010, J. Stat. Mech., P06019. 
[44] Monchaux R, Cortet P.-P, Chavanis P.-H, Chiffaudel A, Daviaud F, Diribarne P and Dubrulle B, 2008, Phys. Rev. Lett., 101174502.

[45] Leprovost N, Dubrulle B and Chavanis P.-H, 2005, Phys. Rev. E, 71036311.

[46] Leprovost N, Dubrulle B and Chavanis P.-H, 2006, Phys. Rev. E, 7346308.

[47] Naso A, Monchaux R, Chavanis P.-H and Dubrulle B, 2010, Phys. Rev. E, 81066318.

[48] Michel J and Robert R, 1994, J. Stat. Phys., 77645.

[49] Turkington B, Majda A, Haven K and DiBattista M, 2001, Proc. Natl. Acad. Sci. U.S.A., 98 12346.

[50] Bouchet F and Sommeria J, 2002, J. Fluid Mech., 464165.

[51] Chavanis P.-H, 2005, Physica D, 200257.

[52] Salmon R, Holloway G and Hendershott M, 1976, J. Fluid Mech., 75691.

[53] Frederiksen J and Sawford B, 1980, J. Atmos. Sci., 37717.

[54] Herring J, 1977, J. Atmos. Sci., 341731.

[55] Merryfield W. J and Holloway G, 1996, J. Fluid Mech., 30985.

[56] Verkley W. T. M and Lynch P, 2009, J. Atmos. Sci., 662216.

[57] Kazantsev E, Sommeria J and Verron J, 1998, J. Phys. Oceanogr., 281017.

[58] Polyakov I, 2001, J. Phys. Oceanogr., 312255.

[59] DiBattista M and Majda A, 1999, Proc. Natl. Acad. Sci. U.S.A., 966009.

[60] DiBattista M and Majda A, 2000, J. Phys. Oceanogr., 301325.

[61] Venaille A. Mélange et circulation océanique: une approche par la physique statistique. $\mathrm{PhD}$ thesis, Université Joseph Fourier, 2008.

[62] Venaille A and Bouchet F, arXiv:1011.2556.

[63] Naso A, Chavanis P.-H and Dubrulle B, 2011, Eur. Phys. J. B, 80493.

[64] Herbert C, Dubrulle B, Chavanis P. H and Paillard D, 2012, Phys. Rev. E, in press arXiv: $1204.5348 \mathrm{v} 1]$.

[65] Naso A, Chavanis P.-H and Dubrulle B, 2010, Eur. Phys. J. B, 77187.

[66] Gradshteyn I. S and Ryzhik I. M, 1980, Table of Integrals, Series, and Products. Academic Press.

[67] Pedlosky J, 1987, Geophysical Fluid Dynamics. Springer.

[68] Jaynes E, 1957, Phys. Rev., 106620.

[69] Balian R, 1991, From Microphysics to Macrophysics. Springer-Verlag, New York.

[70] Balian R, 2005, Studies in History and Philosophy of Modern Physics, 36323.

[71] Jung S, Morrison P and Swinney H, 2006, J. Fluid Mech., 554433.

[72] Turkington B and Whitaker N, 1996, SIAM Journal on Scientific Computing, 171414.

[73] Robert R and Sommeria J, 1992, Phys. Rev. Lett., 692776.

[74] Chavanis P.-H, 2009, Eur. Phys. J. B, 7073.

[75] Chavanis P.-H and Sommeria J, 1996, J. Fluid Mech., 314267.

[76] Bretherton F and Haidvogel D, 1976, J. Fluid Mech., 78129.

[77] Matthaeus W and Montgomery D, 1980, Ann. N. Y. Acad. Sci., 357203.

[78] Leith C, 1984, Phys. Fluids, 271388.

[79] Batchelor G, 1969, Phys. Fluids, 12 (Suppl. II) 233.

[80] Chavanis P.-H and Sommeria J, 1998, J. Fluid Mech., 356259.

[81] Brands H, Chavanis P.-H, Pasmanter R and Sommeria J, 1999, Phys. Fluids, 113465.

[82] Bouchet F, 2008, Physica D, 2371976.

[83] Chavanis P.-H, 2008, Physica D, 2371998.

[84] Chavanis P.-H, Naso A and Dubrulle B, 2010, Eur. Phys. J. B, 77167.

[85] Fofonoff N. P, 1954, J. Mar. Res., 13, 254.

[86] Touchette H, Ellis R. S and Turkington B, 2004, Physica A, 340138.

[87] Rockafellar R, 1970, Convex Analysis. Princeton University Press.

[88] Lynden-Bell D and Wood R, 1968, Mon. Not. R. Astron. Soc., 138495.

[89] Thirring W, 1970, Z. Physik., 235339.

[90] Barré J, Mukamel D and Ruffo S, 2001, Phys. Rev. Lett., 8730601. 
[91] Zinn-Justin J, 2002, Quantum Field Theory and Critical Phenomena. Oxford University Press.

[92] Bouchet F and Corvellec M, 2010, J. Stat. Mech., 2010 P08021.

[93] Corvellec M. Transitions de phase en turbulence bidimensionnelle et géophysique. PhD thesis, ENS Lyon, 2012.

[94] McWilliams J. C, 1990, Phys. Fluids A, 2547.

[95] McWilliams J. C, 1990, J. Fluid Mech., 219361.

[96] Brachet M, Meneguzzi M and Sulem P, 1986, Phys. Rev. Lett., 57683.

[97] Brachet M, Meneguzzi M, Politano H and Sulem P, 1988, J. Fluid Mech., 194333.

[98] Benzi R, Paladin G, Patarnello S, Santangelo P and Vulpiani A, 1986, J. Phys. A, 193771.

[99] Benzi R, Patarnello S and Santangelo P, 1988, J. Phys. A, 211221.

[100] Babiano A, Basdevant C, Legras B and Sadourny R, 1987, J. Fluid Mech., 183379.

[101] Schneider K and Farge M, 2005, Phys. Rev. Lett., 95244502.

[102] Rhines P, 1975, J. Fluid Mech., 69417.

[103] Cho J and Polvani L, 1996, Phys. Fluids, 81531.

[104] Yoden S and Yamada M, 1993, J. Atmos. Sci., 50631.

[105] Williams G, 1978, J. Atmos. Sci., 351399.

[106] Basdevant C, Legras B, Sadourny R and Béland M, 1981, J. Atmos. Sci., 382305.

[107] Fjortoft R, 1953, Tellus, 5225. 